\title{
Quantum Inverse Measurement Theory Contributing to the Birth of Interpretation System of Quantum Mechanics of Local-Realism and Determinism
}

\author{
Runsheng Tu \\ National Special Steel Quality Products Supervision and Inspection Centre, Huangshi, China \\ Email:2run3@sina.com
}

How to cite this paper: Tu, R.S. (2017) Quantum Inverse Measurement Theory Contributing to the Birth of Interpretation System of Quantum Mechanics of LocalRealism and Determinism. Journal of Modern Physics, 8, 1398-1469.

https://doi.org/10.4236/jmp.2017.88088

Received: May 8, 2017

Accepted: July 18, 2017

Published: July 21, 2017

Copyright $\odot 2017$ by author and Scientific Research Publishing Inc. This work is licensed under the Creative Commons Attribution International License (CC BY 4.0).

http://creativecommons.org/licenses/by/4.0/ (c) (i) Open Access

\begin{abstract}
The existing interpretation of quantum mechanics is contrary to common sense. The existing quantum mechanical interpretation schemes are puzzling. The confusing theory is unconvincing, and needs to be amended and completed. The successful interpretation program of quantum mechanics of local-realism and determinism is undoubtedly the most attractive. Preparing the interpretation program deserves to be chosen as a research goal. It is a very good premise to believe that an object particle consists of light-knot of monochromatic waves. According to this premise, the erroneous recognition about "superposition principle, wave-particle duality and uncertainty principle" can be corrected. Under this premise, above research goal is achieved by establishing, applying quantum mechanics inverse measurement theory, adhering to the principle that there must be a complete empirical chain in the derivation process of experimental conclusion, and using the side effect caused by accompanying-light to explain the diffraction experiment of object particles. Electron secondarily diffraction and other experiments directly prove that there is the measurement (observation) which may not destroy quantum coherence. The diffraction experiments of all kinds of particles show that the Keeping and playing of the coherence of moving particles in the vacuum have nothing to do with their previous experience. These are the existing experiments, to be found, that support the theory of quantum inverse measurements. The verification experiment of quantum inverse measurement is designed. The absolute superiorities of quantum inverse measurement and the new view of measurement of quantum mechanics are listed. These superiorities are that: it has the characteristics of local-realism and determinism; it is not contrary to common sense and there is no confusing place; it can predict several phenomena that cannot be predicted by other theories. A solid
\end{abstract}


theoretical foundation has been laid for "correctly understanding the microscopic world" and establishment of local realism quantum mechanics.

\section{Keywords}

Quantum Inverse Measurement, Local Realism, Determinism, Quantum Entanglement, Tu's Measurement View of Quantum Mechanics, Light-Knot Electronic Structure Model

\section{Introduction}

Quantum mechanics can be divided into several components: the mathematical formal system of quantum mechanics, the interpretation system of quantum mechanics (the most important is quantum mechanics measurement view) and quantum mechanics philosophy view. The mathematical form system of quantum mechanics can be divided into: Theoretical premise part (quantum mechanics postulate), logical inference part and conclusion part. The successful application of quantum mechanics shows that its mathematical logical conclusions are available. However, the postulates of quantum mechanics, the interpretation and the philosophical view of quantum mechanics are puzzling (confusing). In this case, it is necessary to optimize the postulations of quantum mechanics and improve the interpretation of quantum mechanics. The composition program of quantum mechanics postulations has more than one. This indicates that it has room for optimization. The author has reduced the 5 quantum mechanical postulates to 1 in the book of "local realism quantum mechanics" [1], and retains the successful part of quantum mechanics (the brief introduction of method of reducing the quantum mechanics postulate is shown in Section 6.2 of this paper). The relation between the interpretation system of quantum mechanics and the mathematical formal system is desalinated by the result which does not require the third postulate of quantum mechanics to be related to the measurement definition and probability generation. It makes the measurement view and mathematical formalism of quantum mechanics change from strong correlation to weak correlation, and the successful application of quantum mechanics is not a valid proof of the correct evidence of the interpretation system of quantum mechanical. QIMT is only to reform the premise of quantum mechanics, interpretation system of quantum mechanics and the philosophical view of quantum mechanics, and does not deny the part of its success. There is no contradiction between QIMT and quantum mechanics logic system. In a word, QIMT criticizes the interpretation system of quantum mechanics without denying the mathematical formal system of quantum mechanics. In other words, we only deny some qualitative explanations of quantum mechanics rather than deny the quantitative conclusion of quantum mechanics. In this way, it cannot deny QIMT that the correctness of the quantitative descriptions of quantum mechanics verified by numerous experimental facts. In fact, "quantum mechanics verified by 
experimental facts" means "validating the quantitative conclusions of quantum mechanics (the conclusion from the mathematical formal system of quantum theory) rather than the qualitative interpretation in the strict sense".

Quantum mechanics has been very successful in applications, but the interpretation of quantum mechanics has been puzzling. The famous Steven Weinberg also wrote that quantum mechanics is confusing (Steven Weinberg, 2017). In addition, quantum mechanics has many different competing interpretations, showing a "quantum mechanical interpretation jungle" of the chaotic state.

Although Copenhagen's explanation can make out a good case, the cost of paying is, the wave function is no longer a completely objective existence, but rather becomes something that depends on the observer. In addition, from the perspective of theoretical completeness, one of the drawbacks of the Copenhagen interpretation is that it needs to presuppose the existence of objects (measuring instruments or observers) described by classical mechanics, and cannot completely derive all the results from the quantum mechanics itself. This leads to it difficult to apply to quantum cosmology so that there is no "observer" or any classical object in principle. Since Copenhagen explanation has a problem, other explanations will birth. Multi-world theory is one of them. Multi world theory can avoid some problems in Copenhagen's explanation, but there are other problems. There are still some questions and controversies about how to explain the probability phenomena in quantum experiments in the multi world theory: since each possibility has been achieved, how to talk about the odds? In the description of quantum theory, this probability comes from which of the many possible worlds is random. In the multi-world interpretation, each time a small interaction will produce a huge number, almost the same parallel to the universe, and we cannot help but feel weird for this. There are also questions about how to understand the so-called "multiple worlds" and whether these parallel universes "really exist". Multi-world theory is like human ancestors imagined the underworld, human world and heaven of the three worlds when they cannot explain the natural phenomenon. The phenomenon can be explained, but cannot confirm the existence of more worlds. Someone claimed that the phenomenon of continuous entanglement was detected. A person observes the phenomenon of continuous entanglement is the same person observed the phenomenon in different worlds. This is to deny the existence of the Multi-world. For the existence of more worlds, the affirmative experimental evidence is not found, but the evidence of denial has been found. The shortcomings of other explanations are not enumerated. There are many kinds of quantum mechanics interpretation, but they are built on the premise that microscopic particles have spooky characteristics, and all of them have not solved the problem of ontology of quantum mechanics (we should know, the confusion of quantum mechanics mainly derived from the ontology of quantum mechanics). It is a fundamental explanation that microscopic particles behave like spooks. As long as the basic explanation is incorrect, the different explanations put forward on this basis are wrong. One of the advantages of QIMT is that it never explains the behavior of micro particles 
into spooky behavior.

Among the existing quantum mechanical interpretation, only one explanation is correct. It is more likely to be incorrect that they are all not correct. Because, their common drawback is that they are non local-realism or non-determinism, to be contrary to common sense and confusing. In other words, their common drawback is that they cannot be separated from the spooks. Copenhagen explanation has to admit that microscopic particles had ghost characteristics. Multi-world theory has to admit that the universe has a spooky character. Implicit parameter interpretation has to admit that the interaction has a spooky character. Confusing and contradictory are unsatisfactory, no charm at all, and it needs to be improved and perfected. The incorrect interpretation of quantum mechanics can lead to quantum mechanics and even human knowledge develops in the wrong direction. Don't cares about its unsatisfactory situation is just an optimistic attitude, rather than the unreasonable things in quantum mechanics do not exist. A theory or idea, once it is admitted that consciousness can affect the behavior of natural things, and there must be a significant lack of understanding. After the establishment of the interpretation system with the advantage of "the problem where the wave function to be from has been solved by means of the model of the kink of the waves forms the particles", this is all the more so. Now, it is a bad sign that those physicists today who are most comfortable with quantum mechanics do not agree with one another about what quantum mechanics all means. The dispute arises chiefly regarding the nature of measurement in quantum mechanics (Steven Weinberg, 2017).

As mentioned above, it is meaningful to explore a satisfactory explanation of quantum mechanics.

The initial motivation of this article is to solve the problem of the development of quantum weak measurement theory (i.e., to solve the measurement problems of quantum mechanics). With the deepening of exploration, I found the existence of events of quantum inverse measurement. And then the function of quantum inverse measurement is found, and quantum mechanics inverse measurement theory (QIMT) is established. And then later, it is to find out the influence of QIMT on the existing quantum mechanics interpretation system. Finally, it is to establish a new interpretation system of quantum mechanics. That is to say, the motive of exploration has gradually developed into the establishment of QIMT and the correction of the misunderstanding of the microscopic world (which is part of the "understanding of the composition, structure and nature of microscopic particles"). The most attractive quantum mechanics interpretation system is scientific, logical, and does not violate the common. I long ago had such a desire to build the most attractive interpretation system of quantum mechanics to solve the problem of quantum mechanics. It is necessary to establish the quantum mechanics of local realism. However, the Ref. [1] did not solve the problem of quantum mechanics interpretation well. Ref. [1] makes up the deficiencies for my previous research work. This paper is complementary to Ref. [1] and constitutes a complete new quantum theory. For the sake of 
convenience, the most attractive measurement view of quantum mechanics established by me-the measurement view of quantum mechanics of local-realism and determinism will be called “Tu's measurement view of quantum mechanics". It is not difficult to establish Tu's interpretation system of quantum mechanics. As long as we adhere to the principle that the empirical chain must be complete, use accompanying-light effects to explain the diffraction experiment by double-slit of electrons, and wake up and find everything changed.

The very important accompanying-light effect is not entirely imagined, but there are experimental basis (for example, the experiment that Masatoshi Koshiba captures neutrino). Neutrinos react with the nuclei of hydrogen and oxygen in water and produce an electron. This electron can cause a faint flash of light. The light also belongs to the accompanying-light. What can be seen in the cathode ray experiment is the accompanying light (Figure 1). The electron beam companion-light in Figure 1 is visible. There is likely to be invisible shortwave companion-light. Who can guarantee that the electron beam used to make the electron diffraction experiment does not produce a companion-light similar to that in Figure 1? After you see the companying light in Figure 1, you will soon realize that there was a major scientific mistake in the past: In the case of that companion-light effect has not been ruled out, we use wave-particle duality to explain electron diffraction experiment. As can be seen from Figure 1, the diffraction is more likely to be caused by companion light. This requires an experimental method to test. We should be curious about the consequences of "diffraction to be caused by companying light".

At the end of 1980s, Aharonov Y. et al proposed the theory of weak measurement [2]. Quantum weak measurement theory is used to measure the signal as weak as possible. So make the interference of the instrument to the measured object as little as possible. The application of the theory has solved a series of problems which cannot be explained by standard measurement theory, and the

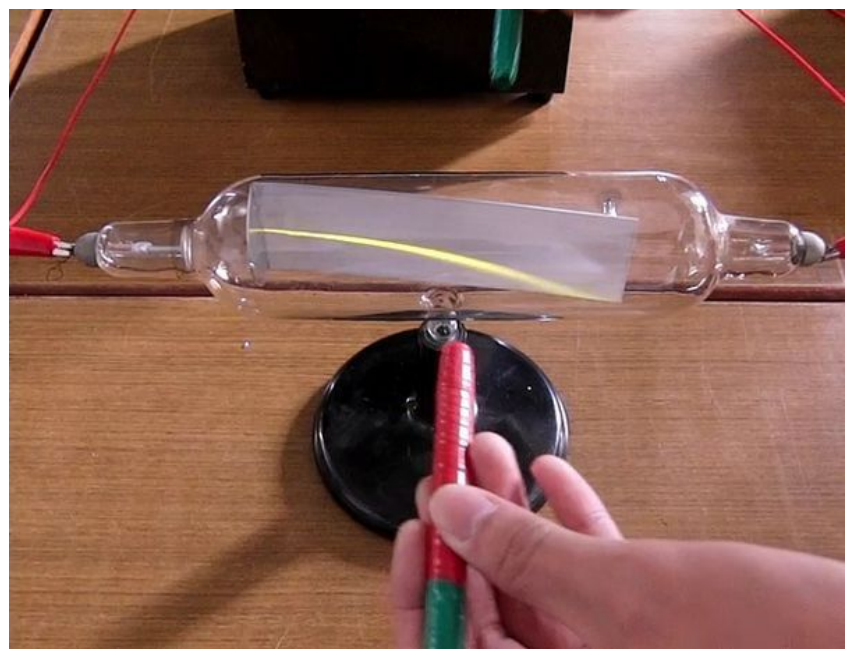

Figure 1. The cathode ray deflected in the magnetic field. In this figure, the two-color stick in the left hand is a magnet, and the luminous rays in a transparent glass tube are electron beam. Cathode ray itself is not visible. We see is its accompanying-light. 
understanding of the basic problems in quantum mechanics is given a relatively clear image. As mentioned above, the ideal quantum inverse measurement is the measurement of the measured particle only sends information to the instrument, and the observer (or instrument) does not send any information to the measured particle (the positive-going signal interference can be ignored). Scilicet, the measurement that the influence (interference) of the observer or instrument on the measured particle can also be ignored is belonging to inverse measurement. Although the observer has an effect on the observed object but it does not affect the part of the observer who wants to see it. This observation is also a partial inverse measure. It is the extension of the concept of quantum inverse measurement that the measurement to be evenly or very symmetrically influencing the measured objects also belongs to quantum inverse measurement. That is, QIMT believes that if the measured particles are affected by the equilibrium (uniform order or very symmetrical), an objective state can also be obtained. Quantum strong measurement and quantum weak measurement generally refers to the measurement that the information is sent by the measuring instrument has an effect on the measured object (destroying its original state: effective interference) [3]. Just the intensity of interference is different. In the direction of information transfer, they are opposite to the quantum inverse measurement. The connotation of the concept of quantum inverse-measurement is the measurement that there is only reverse signal transmission or action. Its extension is the measurement that the impact of the environment on the measured object can be neglected.

The development model of quantum weak measurement theory is the measurement using as weak as possible signal, or using more and weaker signal". However, the measured signal is weak to a certain extent cannot be measured. Therefore, this development idea is a dead end. We must about this question: Can we use other ways to achieve the ideal that can get a pure objective measurement results? All observation or measurement is achieved through the transmission of the signal, and the signal transmission has a positive and inverse two directions: A positive signal is transmitted from the observer to the observed object; the reverse signal is transmitted from the observed object to the observer. If only the signal from the observed object to the observer, the observation and measurement are also achievable. In the macro world and real life, there are a large number of such measurements (and/or observations). For example, in the night, we observe the signal bomb (tracer). According to the whistle and determine the approximate location of travel vehicle; according to their friends or family, the blind judge their position and identity; the snake measures the position of the prey object... That is to say, the measurement does not necessarily have to be like a bat to prey on the need for two-way signal transmission and mutual influence. It is also possible that only the measured object (or the observed object) adversely affects the observer (this type of measurement is called inverse measurement). From a logical perspective, in the microscopic world, the 
measurement that only there is signal reverse transmission is the measurement that has not any disturbance to the observation object and that cannot lead to wave packet collapse. Such measurements are the ideal measurement that can obtain purely objective results. The development and ultimate goal of quantum weak measurement theory is to realize the interference-free measurement.

In the course of any measurement, the effective process is that the observer receives the information from the observed object. This information is not necessarily the feedback information from the observer. It can be just the reverse information that is sent by the observed object. It does not meet the logic that pure objective observation results to cannot be obtained by interference-free measurement. Nowadays, there are a number of people who acknowledge the existence of protective measurement. Quantum inverse measurement belongs to the category of quantum protective measurement. The rest of the question is "whether there is the measurement without positive interference". This article will demonstrate this critical issue. In this paper, the measurement that the instrument does not interfere with the measured object (or the measurement that the interference intensity is less than the anti-interference ability (robustness), interference can be ignored, and may not lead to wave packet collapse) is called the measurement without positive interference, inverse measurement (or interference-free measurement) for short.

The author introduces his research motivation at the beginning. The starting point and basic principle of QIMT are introduced in Section 3. In the following chapter, the conclusion, the case, the prediction and the verification method of QIMT are introduced. Especially QIMT to be important influence on superposition principle, uncertainty principle and the concept of wave-particle duality is introduced in detail. In the section 8 , we will design the principle of the several experiments: electron diffraction experiments in cloud chamber, in electric field and/or special medium. These kinds of experiments can judge the measurement view and the interpretation system of quantum mechanics. To combine them together, the effect is better. One of the most important contents of this paperthe advantage of QIMT and the deficiency of other similar theories will be introduced in Section 7.

The definition of measurement by quantum mechanics is a hypothesis - the third postulate of quantum mechanics. The concise expression for this definition is that when a physical system is in state $|a\rangle$ and a physical quantity $Q$ is measured, the expected value is $\langle Q\rangle=\langle a|Q| a\rangle$. It is misinterpreted as: only the operation that can cause the quantum state to change is quantum measurement. This kind of misinterpretation exists in a series of questions. First, why do not we consider the positivism effect of the operation with a positivism function other than quantum measurement? That is, why should we exclude the measurements that do not lead to change in state? Second, is the quantum state change and quantum decoherence always synchronized? If you think they're synchronous, what's the reason? Under the premise that they are not synchronized, if the quantum state changes but decoherence does not occur (or decohe- 
rence occurs and quantum state do not changes), how do we handle these operations? In fact, no matter how we define measurement, we can't change this fact that: In the process of measurement, the transmission and function of information and matter can be divided into two cases of one-way or two-way. The one-way can be divided into two kinds of reverse and forward. "Information transfer" is the soul of measurement, and "empirical function" is the basic requirement for measurement. State change is neither the essence of measurement nor the basic requirement for measurement. If, as long as the measurement, a quantum state change is made, then the quantum measurement cannot have an empirical effect on the pure objective quantum state. Empirical needs to be perceived, while perception requires interaction or transmission of information. Quantum measurement has been understood as "spiritual communication" by existing quantum scientists. This is clearly contrary to the spirit of science. The measurement I define is a set operation that the empirical function determined by the real interaction. Quantum inverse measurement is the measurement that there is only the reverse signal transmission or the effect of a positive signal on the measured object is negligible. The following is used interaction as an element to define a measurement with an empirical function.

Measure object: the object being measured or observed, also called the object of measurement. Abbreviation: target object. Measuring instrument: artificial environmental substances that can be affected by the target object and/or the artificial environment substances that target substances are affected by them It can also be called observer. Measurement: the process of unidirectional or bidirectional transmission of information (or there is one-way or two-way interaction) between the target and the instrument. A more complex measurement can be divided into several local measurements. Human participation is not a necessary condition for measurement. People play two roles in measurement: one is to design the measurement process and implement the measurement operation; the two is to collect and analyze the information obtained from the measurement. Observation is the way of one of measurements. The classification of the measurement is shown in the following figure:

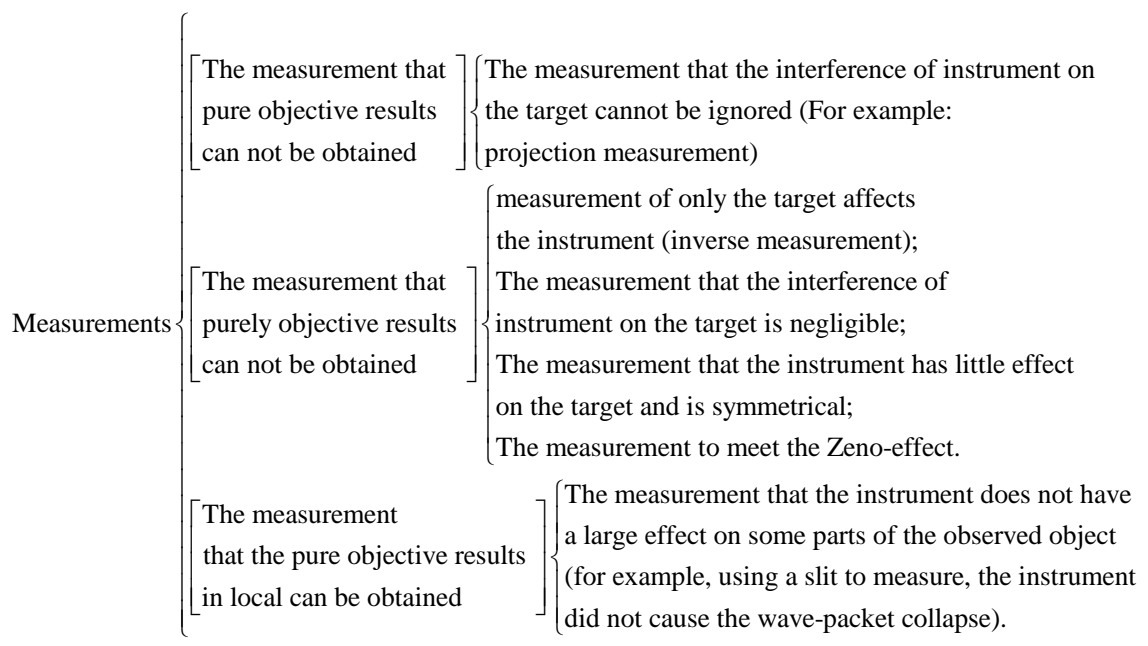




\section{The Very Serious Logical Questions in the Interpretation System of Existing Quantum Mechanics}

The problems described in this section are the serious problems that exist in quantum mechanics. In other chapters, these questions may be discussed. Other chapters also discuss other issues of quantum mechanical interpretation. The existence of these problems fully indicates that the existing interpretation system of quantum mechanics is incomplete. If we do not care about these contradictory, we still want the spirit of science? The existence of these questions suggests that the other explanations that are less problematic are worth discussing.

Can the measurement cause the packet to collapse? One is a hypothesis, the other is experimental fact, and we should first choose which one? For quantum physicists, these two questions seem to be difficult to answer. However, at present, there is a big problem with their choice. For example, they chose to prioritize the assumptions, and they sometimes use the idea that measurements did not cause the waves to collapse.

\subsection{Does the Measurement Lead to Wave Packet Collapse and Quantum Decoherence Inevitability?}

Quantum physicists have chosen the conclusion that "as long as measurements, wave packet collapse and quantum decoherence will be caused". For example: the use the cloud chamber, spark chamber and other equipments to measure micro particles (see Section 4.3 for details); a quantum state is measured for the first time in a quantum entanglement experiment. The existing interpretation system of quantum mechanical also requires this choice. But on some occasions, they secretly chose the conclusion that measurements would not cause wave packet collapse (i.e., they were not consistent. For example, when quantum entanglement is proved to exist, they did so). That is to say, their actions are very contradictory.

In addition, many experiments and facts show those quanta decoherence and wave packet collapse cannot be caused by measurements (or it is proved that the wave packet collapse process does not exist by experiments). These experiments are as follows.

In the experiment of microscopic particle diffraction, when a particle beam passes through the slit, the influence of the slit on the particle beam belongs to the influence of the instrument on the measured object. However, the undulatory property of the particles passing through the slit not only does not disappear, but show undulatory property in the process of penetration. The charged particles are subjected to the action of strong electric field and the collimation of the magnetic field before passing through the slit. These actions are in line with the definition of measurement (observation). None of them caused the wave packet to collapse (otherwise we can't see the diffraction pattern). This is the experimental fact that measurements do not cause wave packet collapse. The secondary diffraction phenomenon of electrons more accurately shows that the front and rear two slit (especially the first) did not cause the wave packet to collapse (it 
can be said that the slits in all diffraction experiments did not cause the wave packet to collapse. The reason is that the collapse occurred on the screen). Double slit diffraction experiments of photons and electrons show that only there is the superposition between measured particles, and the superposition between particles and instruments do not occur. It can be seen that the doubleslit diffraction experiment of electrons and the secondary diffraction experiments of electrons show that the measuring instrument does not cause the essential properties of the measured particles to change. In the other words, the purely objective properties of the measured particles are presented by these measurements, rather than the purely objective properties of the measured particles ate changed by these measurements. The above facts can be explained as part of the measurement cannot lead to wave packet collapse and quantum decoherence, but also can be interpreted as wave packet collapse process and quantum decoherence process does not exist. If the measurements must lead to a superposition of the states between the instrument and the measured particles, a small piece of optical fiber can only transmit signals that are seriously distorted. The fact is that a long fiber can transmit undistorted signals. In a sense, anti distortion technology is also a technique to recover the quantum coherence of signal carrier. The fact that the state superposition between the instrument and the measured particles are not necessarily, with the instrument measurement may see the pure objective of the performance of particles. In this way, it is possible to achieve the quantum inverse measurement mentioned above (spying on purely objective properties of microscopic particles). Some people claim to observe the continued entanglement of particles. Logically, only the measurement (observation) did not lead to quantum decoherence, the phenomenon of continuous entanglement may be observed. Therefore, the experiments that have observed continuous entanglement of particles have proved that the measurement may not lead to quantum decoherence. Some experiments have been done to keep the ions stationary in the microcavity while maintaining the coherence characteristics of the ions. This experiment also shows that the measurement operation of the controlling ions did not lead to the disappearance of coherence.

If we adhere to the principle of science, we must choose between "the measurement will inevitably lead to wave packet collapse and quanta decoherence" and "measurement may not lead to wave packet collapse and quantum decoherence". If the former is chosen, the experimental facts are violated. If the latter is chosen, first, the existing concepts of quantum mechanics are eliminated, and secondly, the conditions for maintaining and destroying quantum coherence must be discussed. The existing quantum mechanics does not discuss the conditions for maintaining and destroying the quantum coherence, but chooses two diametrically opposite views subjectively and alternately according to their own needs. So, at this point, quantum physicists make ordinary readers confused (they themselves are confused). In fact, existing interpretation systems of quantum mechanics will disintegrate as long as measurements do not lead to wave packet collapse and quantum decoherence. As long as it is proved that there is 
the experiment which may not destroy the quantum coherence, it is proved that the quantum inverse measurement experiment can be realized.

\subsection{Should We Believe in Hypothesis or Should We Believe in Experimental Facts?}

Photon and electron diffraction experiments can also be said that the use slit to measure photons and electrons. However, the most part of the process ahead this measurement did not cause the wave packet to collapse; otherwise we would not see the diffraction pattern. In the experiment to have claimed seize the Schrödinger's cat of dead-live hybrid, the persistent entanglement was observed by continuous measurements. If this is true, it also shows that measurements do not result in wave packet collapse. If the measurement will lead to wave packet collapse, the experimenter cannot see the continuity of quantum entanglement, only to see the quantum entanglement stopped at the beginning of the measurement. Photons through glass also belong to use glass as an instrument to measure photons. When the photons smoothly through a glass, state superposition did not occur and the photon did not change into a pure particle without volatility.

Section 2.1 lists the experiments that can prove that wave packet collapse does not exist or does not occur. Both the collapse of wave packet and the related superposition of quantum states are only theoretical hypotheses. Which one should we believe? At present, quantum physicists have believed the hypothesis and not the facts. These ideas have solidified in his mind. Many people willingly are unscientific, but also to maintain their established ideas (of course, some people don't do it consciously).

The concept that interference-free measurement can also change quantum states has not any experimental basis, there is no reliable theoretical basis, is not consistent with the logic, and does not belong to the category of natural scientific concepts. It is a kind of philosophical view (or is illogical belief). QIMT points out that, for want to get the measurement results of determined and purely objective, the measurement under the condition of only reverse influence is equivalent to interference-free measurement. The establishment of QIMT breaks through this concept and the concept that interference cannot be eliminated (the old view of measurement of quantum mechanics), is a conceptual progress, and is a leap of human knowledge.

Maybe someone will ask that the existence of quantum entanglement and the experimental results of double slit diffraction are the two obstacles to QIMT, and what does the author think about this problem? Here I want to tell everyone that quantum entanglement experiments did not use the experimental method to exclude that the twin particles are all pigeon pair when they born. The sex of pigeon pair alternating is still the pigeon pair, rather than the mixture of boy and girl. We have no reason to say that the experimental method has been used to confirm the existence of quantum entanglement. As already mentioned, the electron secondary diffraction experiment can also prove that the general measurement 
does not lead to wave packet collapse, and the quantum inverse measurements can be achieved. Only the possibility is excluded that the diffraction in the diffraction experiment by double-slit is caused by accompanying light, can we say that this kind of experiment confirms the existence of the wave-particle duality and the non-local particle. It is recognized that the physical particles such as electrons are not composed of wave packets and can be made of monochromatic waves. It is concluded that the effective superposition of states is limited by the spatial distance and the direction of the force. See Section 5 for more and more detailed description.

\subsection{If the Function of the Instrument Can Cause Decoherence, Then How Do Coherent Particles Come from the Instrument?}

Why is a change in the state of a measured particle necessarily caused by the measurement that the association between the instrument and the measured particles has not occurs (one that quantum decoherence has not occurs)? Does quantum state change always occur simultaneously with quantum decoherence and wave packet collapse? If it is not, what should I do?

Does the instrument in the end produce quantum coherence or damage quantum coherence? Since the particles are produced by the instrument, the quantum coherence of the particles is breaded by the instrument. This sentence says that the instrument can nurture quantum coherence. The Copenhagen school believes that as long as the measure, the observer (or instrument) will destroy the quantum coherent state. This sentence says that the role of the instrument must destroy the quantum coherence. Particles with quantum coherence are all born out of the instrument. The studied particles are made out of instruments, not out of thin air. Even cosmic radiation, it also comes from the super instruments of the universe. It is an experimental fact that an apparatus for breeding particles does not cause decoherence. If the effect of the instrument would lead to quantum decoherence, then particles with quantum coherence would never be created. Conversely, if the instrument can produce particles with quantum coherence, the instrument may not destroy the quantum coherence. It is obvious that the viewpoint that the instrument can destroy the quantum coherence is contradictory to the experimental facts. This is also one of the most serious contradictions that exist in the interpretation system of existing quantum mechanics.

The two electron diffraction experiment shows that the slit has led to the change of the electron state. However, the first slit does not result in the disappearance of the quantum properties of the incident electrons (does not cause wave packet collapse and quantum decoherence). This indicates that the measurement of quantum states and quantum decoherence can occur at different times. The association between the instrument and the measured particles may also not occur. As long as there is the case where the state superposition between the instrument and the measured particle does not occur, we can discover the purely objective and determined quantum properties of the particle by mea- 
surement. If you insist that the measurement will inevitably lead to changes in the quantum state, it produced a question: Why is a change in the state of a measured particle necessarily caused by the measurement that the association between the instrument and the measured particles has not occurs (one that quantum decoherence has not occurs)? "In some quantum-measurement process, the association between the measured particles and the instrument does not occur" is an empirical point of view. It has not verified that the association between the measured particles and the instrument are inevitable in all quantum measurements. Which view should we believe?

\subsection{Does the Instrument That Gives Birth to Particles Protect "Is Child" with Motherly Love? Is the State of the Particle Prior to Measurement Obtained by Extrapolation Reliable?}

Before and after two measurements of the polarization of the same photon, the result is that the polarization direction of the photon has not changed", i.e., "the measurement does not cause the quantum state of the photon to change". The spin direction of the same electron is measured two times before and after, and it can be also found that the spin direction of the electron has not changed due to measurements. This kind of experiment can be repeated many times. It can be said that "the measurement does not lead to changes in the quantum state" has been empirical. Now, on the basis of the above empirical results, we deduce whether the first measurement will lead to a change in the quantum state. The rigorous deductive result is also the first measurement and will not lead to a change in the quantum state. However, the interpretation of orthodox quantum scientists by means of deduction is that the first measurement can lead to changes in the state, and the subsequent measurements will not lead to state changes. Such a conclusion does not accord with deductive logic on the one hand, but on the other hand, it runs counter to the deductive way of obtaining the conclusion of uncertainty. The reason they use this deductive logic is that the measurements after the first measurement are not quantum measurements again, but classical mechanical measurements. Such a sophistry leads to an obvious problem that is difficult to answer is to measure a photon, how to make a photon, under the circumstances of that both its morphological features and mode of motion are invariant, into a classical mechanical particle by means of measurement? The same is true for electrons: electrons that fly in a vacuum that is almost unaffected by an external field. Why say it is the classic particles? You know, before the measurement, it is also impossible that the particle is not affected by the instrument of breeding it (Section 2.3). Unless the instrument that gives birth to particles can take care of its child with motherly love, the laws for the effect of all instruments on the particles should be the same.

Since it is believed that the measurement will change the state of the particle, the state of the particle before the measurement cannot be verified by experiment. Quantum scientists use a deductive method to obtain the states of particles before measurements (or when they are not measured). The diffraction 
experiments of electron and other particles prove that the particles in measurement are uncertain. Thus, scientists also deduce the conclusion that particles are also uncertain when they are not measured. Affected by the measuring instruments and not affected by the measuring instruments, the two conditions are not the same. From the affected by measuring instrument extrapolated to not affected by the measuring instrument is not a deduction, but a guess.

\subsection{Does the Quantum Entanglement Disappear When Measured or Not Exist Originally? How to Exclude the Concept That Quantum Entangled State Would Do Not Exist Originally"}

There are other problems that quantum mechanics has not solved. For example, what is the basis of the necessity of state superposition? How to overcome the contradiction between the interpretation of instantaneous quantum entanglement experiment and the interpretation of continuous quantum entanglement experiment? And so on.

The existence of quantum entangled states is a hypothesis. When we use the instantaneous quantum entanglement experiment to prove the authenticity of this hypothesis, the other hypothesis that measurements inevitably eliminate entanglement state is necessary. The general result is to use the assumption of entanglement elimination to verify the assumption that an entangled state exists. This is a very funny logical cycle. This logical cycle simply cannot prove the tangent state of the real existence.

For experiments that prove persistent entanglement, the interpretation is based on the fact that measurements do not cause entanglement to vanish". This is contrary to the idea that "measurements can lead to disappearance of entangled states" to be used by instantaneous quantum entanglement experiments (there is a contradiction between the two). What kind of beliefs should we believe? This kind of contradiction determines that the existing quantum entanglement experiments cannot prove the existence of quantum entangled states without ambiguity.

As mentioned above, the destruction of the original state of a measured particle by an observer (instrument) is imaginary, without empirical evidence. This makes the interpretation for quantum entanglement extremely unreliable (Because the existence to verify quantum entanglement must depend on that measurement can lead to the change in the state). The existence of entangled states and other superposition states before measurement of particle, there is neither a solid theoretical foundation nor a solid experimental basis. It is still just a hypothesis that is imagined. No conditions of superposition (entanglement) without the limitations of distance are absurd. "The experimental facts have proved the existence of quantum entangled states," said the remark too early.

\section{The Scientific Basis of Quantum Inverse-Measurement Theory}

The basis of the quantum inverse measurement theory discussed in this section 
can be regarded as a starting point. The Section 3.1 together with Section 6.2 can form a solid foundation for QIMT.

In the world of classical mechanics, there is a measurement that only the signal is sent by a measured object and the signal only is received the measuring instrument and the measured object is undisturbed. In the micro world, this form of measurement is also an objective reality. The theoretical basis of quantum inverse measurement is that there is interference-free measurement in which the measured object sends only signals while the measuring instruments only receive the signals. In this measurement, the measured object is the same as the isolated object in the vacuum. The evolution of the state of the measured object can still be described by the Schrodinger equation. All experimental facts show that the keeping up and bringing into play of the diffraction properties of the electrons moving in the vacuum are independent of their previous experience. It is possible to find the experimental facts that measurements do not cause coherence to disappear. This suggests that the inverse measurements that interference can be neglected can also be found. According to the "irrelevance" mentioned above (we consider that: the wave packet collapses as a hypothesis; in electron-diffraction experiment, the electron beam is subjected to the action of a strong electric field and the collimation of a magnetic field), we can inferred out that the electrons passing through the spark chamber must be diffracted. Then the electron beam in the spark chamber does not lose its quantum coherence. If priority is given to experimental facts rather than assumptions, such a conclusion will be accepted-the measurement of utilizing the spark chamber and cloud chamber may be the measurement that the coherence of the measured particle dose not disappear (see Sections 3.1 and 4.3 for details). The uncertainty principle, which hinders quantum inverse measurements to be recognized accepted, has been challenged with unprecedented intensity.

\subsection{Tracing to the Source of Quantum Inverse Measurement}

Quantum weak measurement is the measurement of interference as weak as possible. Quantum weak measurement theory needs to continue to develop. Its ultimate goal must be to achieve interference-free measurement. Quantum inverse measurement is interference-free measurement and/or the measurement that interference can be ignored. This is the ultimate goal of quantum weak measurement, and also a type of nondestructive measurement. Therefore, the source of the theory of quantum inverse measurement is the theory of quantum weak measurement. Both the weakness of quantum weak measurement theory and existing quantum nondestructive measurement theory are that, in the framework of projective measurement, the minimization of interference is minimized by means of weakening signals. The quantum inverse measurement theory breaks through that frame.

To observe the mechanical quantity $\mathrm{A}$, the measured value must be one of its eigenvalue spectra $a_{\mathrm{i}}$. After the observation, the system will be in its corresponding eigenstate $\left|a_{i}\right\rangle$. If the system is also in the eigenstate $\left|a_{i}\right\rangle$ before observa- 
tion (before and after measurement, the state of the system does not change), the measurement result must be a definite value $a_{i}$. This process can be expressed by an eigenfunction equation

$$
\hat{A}\left|a_{i}\right\rangle=a_{i}\left|a_{i}\right\rangle
$$

That is, if the system is in a certain state before the observation, the result of the interference-free measurement is unique. This is determined by the nature of the wave function. It indicates that the measurement leads to a change in the state and the randomness of the measurement results is not necessarily, but conditional. These conditions are: $<1>$ if the state of the system is uncertain before the observation, the obtained eigenvalues after the observation are uncertain; $<2>$ if the interference of measurement cannot be ignored and unpredictable, it conforms to the law of statistics, and the result of measurement is random. Only according to the rules of Bonn (which is based on the uncertainty principle) and that the system state is always uncertain $|\alpha\rangle$ before observation, the system state change to $|\beta\rangle$ a after measurement (observation), and get the eigenvalue of a mechanical quantity $A$, we can consider a measurement will inevitably lead to state change. This procedure can be expressed in the lower form.

$$
\hat{A}|\alpha\rangle=a_{i}|\beta\rangle
$$

However, the situation described in Equation (1) is also very common in quantum mechanics. Such as, $\hat{p}|\psi(x, t)\rangle=p|\psi(x, t)\rangle$ (replacing $\hat{p}$ with other mechanical quantity operator, this formula is also established). For real particles, the description in Equation (2) is hypothetical (for example, it is a hypothesis that measurement necessarily leads to the destruction of superposition states. EPR has assumed an entangled state wave function

$$
\Psi\left(x_{1}, x_{2}\right)=\int_{-\infty}^{+\infty} \exp \left[i p\left(x_{1}-x_{2}+x_{0}\right) / \hbar\right] \mathrm{d} p
$$

It is assumed that the measurement of it will result in the disappearance of the $x_{0}$ entry in the state function. Here, the state function is hypothetical, and state function change is also hypothetical).

Compare of the conditions that Equations (1) and (2) are tenable, it is knowable that, as long as the state before measurement is determined, and the interference can be ignored (measurements do not lead to change of state), it is entirely possible to obtain the determined eigenvalue. Quantum inverse measurement is discussed in the case of Equation (1) applicable. This matter that the state of the system is uncertainty before observation is no and cannot be empirical, always just a hypothesis. The uncertainties at measurement and after measurement are confirmed by electron diffraction and other experiments. The uncertainty before the measurement is the extrapolation of this empirical. You should allow others to doubt this extrapolation. Quantum scientists must also allow others to suspect that measurements are inevitably changing the state of the system.

In the Schrödinger's death-live cat state experiment, we first install an infrared detector probe in the box. People have already acquiesced in that the box and the 
equipments in the box had no effect on the cat's state. Adding a probe that receives only signals, the equipment does not affect the cat's state. The occurrence and continuation of this matter that the cat's body emits infrared signals does not have anything to do with whether the observer is watching or not. The difference between detection and non detection (observation and non observation) is merely the difference in consciousness of the observer. If it is believed that human consciousness cannot directly affect the movements and changes of natural objects, such inverse measurements will not change the quantum states of the measured objects (this procedure can be expressed as $\hat{A}|\alpha\rangle=a_{i}|\alpha\rangle$ or Equation (1)). It is this process that occurs when the charged particles shoot into the cloud chamber before the collision.

If we use $\hat{M}\left(a_{i}\right) \hat{M}\left(b_{j}\right)$ to indicate the order of measurement, the reverse order is $\hat{M}\left(b_{j}\right) \hat{M}\left(a_{i}\right)$, we have

$$
\begin{aligned}
& \hat{M}\left(a_{i}\right) \hat{M}\left(b_{j}\right)|\alpha\rangle=\left|a_{i}\right\rangle\left\langle a_{i} \mid b_{j}\right\rangle\left\langle b_{j} \mid \alpha\right\rangle \\
& \hat{M}\left(b_{j}\right) \hat{M}\left(a_{i}\right)|\alpha\rangle=\left|b_{j}\right\rangle\left\langle b_{j} \mid a_{i}\right\rangle\left\langle a_{i} \mid \alpha\right\rangle
\end{aligned}
$$

This is the operator expression of continuous measurement. Its realization condition is that the measurement does not cause a change in the state. There is no reason why interference-free measurement can cause a change in state. Therefore, interference-free measurement can be carried out continuously. Section 6.2 illustrates why general measurements do not cause change in wave form.

If $<1>$ the two eigenvalues $a_{i}$ and $b_{i}$ of two mechanical quantity $A$ and $B$ of a particle have the common eigenstates $\left|a_{i}\right\rangle=\left|b_{i}\right\rangle$, and $\langle 2\rangle$ the system is in this state, measurement of mechanical quantity $A$ and $B$ is that you can exchange (of order measurement), and can be done at the same time, we have

$$
\begin{gathered}
\hat{M}\left(b_{j}\right) \hat{M}\left(a_{i}\right)=\hat{M}\left(a_{i}\right) \hat{M}\left(b_{j}\right) \\
\hat{M}\left(b_{i}\right) \hat{M}\left(a_{i}\right)\left|a_{i}\right\rangle=\hat{M}\left(a_{i}\right) \hat{M}\left(b_{j}\right)\left|b_{j}\right\rangle=\left|a_{i}\right\rangle=\left|b_{j}\right\rangle
\end{gathered}
$$

The condition that Equation (7) is tenable just is the condition that can be measured at the same time. If one condition is added, $\langle 3\rangle$ the interference of measurement is too weak so that the effect on the state of the system can be neglected, $\hat{M}\left(b_{j}\right) \hat{M}\left(a_{i}\right)=\hat{M}\left(a_{i}\right) \hat{M}\left(b_{j}\right)$ can be changed to

$$
\hat{M}\left(a_{i}\right) \hat{M}\left(a_{i}\right) \approx \hat{M}\left(b_{j}\right) \hat{M}\left(b_{j}\right)
$$

Compare with Equation (7), the condition of Equation (8) to be tenable is the condition that can be measured continuously at the same time. For the measurement that instrument is only affected by the measured object and the instrument does not affect the measured object, the above conditions $<3>$ should be achieved. So that continuous measurement can be realized. Not only can we fail to eliminate the condition $\langle 3\rangle$, but we can also find the proof that the condition $<3>$ has been satisfied: use clouds to capture particles (see Section 4.3).

Believe that the same particle will not appear in two and more than two different places. In particular, do not use the interpretation and ideas of "human consciousness can affect the behavior of micro particles". Determining the states 
superposition allowed by mathematics does not represent the inevitable states superposition in physics. In particular, do not believe that micro particles must be superimposed with their own shadow. Even if quantum superposition occurs, it is not destroyed without touching it (that is, the anti-interference ability of the quantum superposition state is not zero, and it is not allowed to be zero by reality). The facts of the asymmetry of information transfer and interaction influence between instrument and the measured object are ubiquitous. These are the basis of QIMT. In the next section, it will talk about the experimental basis. That is, only the measurement of the information passed from the observed object to the instrument (or the measurement of negligible positive interference, which does not lead to collapse of the wave packet) is real.

We divide the influence of things into three types: the positive influence, the reverse influence and the two-way interactions. Instrument (or observer) unilateral effect on the observed object, the information is transferred from the instrument (or observer) to the observed object; this is the positive influence (or information forward transfer). In contrast to the situation is the inverse influence (information reverse transmission). Both the information (or matter) transferred from the observer to the observed object, and information (or matter) transferred from the observed object to the observer is a two-way interaction. Only the quantum measurement of information reverse transfer is called quantum inverse measurement, other analogies. Please note: the reverse effect is equivalent to that the instrument has no effect on the observed object (or influences can be ignored)! The extension of the quantum inverse measurement concept is the measurement that the effect of the environment on the target could be neglected. If we describe it in the language of quantum mechanics, the quantum inverse measurement is a measure that does not lead to the wave collapse and quantum decoherence. The measurements exist that only there is the reverse influence and no positive influence (or a positive influence can be ignored). There is no reason to deny the existence of such measurements. We can easily find examples of such measurements. The principle, method, basic idea (conclusion), prediction and verification will form QIMT.

Launch a signal bomb (tracer) into the darkness of the night sky, no matter whether the people on the ground with the naked eye to observe it, the movement state of signal bomb will be not affected by observer. The reason is that only the signal transmitted from the observed object to the viewer's eye, there is not any signal transmitted in the opposite direction when an observer observe it with their naked eye (there is not any signal transmitted from the viewer's eye to the observed object). The movement state of the signal bomb does not affected by the observer. When no one is observed, the light emitted by signal bomb is absorbed by the environment. Whether the light signal is absorbed by the environment or by the eyes of the observer, the degree of signal bomb to be interfered by the light receivers is exactly the same. It can be seen that with naked eye to observe signal bomb is the observation of one-way transmission of information. It belongs to the non-interference measurement. In the quantum mechanics 
measurement, there exist the measurement that the information transmit only in one-way.

Orthodox quantum mechanics believe state superposition principle, and believe that microscopic particles can superpose with their own shadow, the homologous conjugated particles are also in the superposition state (entangled state). Hereinafter these superposition states are referred to as the original spontaneous superposition state of the microscopic particles). The form of superposition may be

$$
|\psi\rangle=\sum_{i} c_{i}\left|\psi_{i}\right\rangle
$$

Here, $c_{i}$ is the probability amplitude that the system is in the $\left|\psi_{i}\right\rangle$, and $\left|\psi_{i}\right\rangle$ is the intrinsic state of measured mechanical parameters $\hat{A}$. Its correspondence eigenvalue is $a_{i}$. If the system is in contact with the instrument, the state of the measuring instrument is described by quantum state $|\phi\rangle$. The Hamiltonian of the interaction between the system and the instrument is

$$
H=-g(t) \hat{A} \hat{P}
$$

Here, $g(t)$ is the coupling coefficient between a quantum system and measuring instrument, and $\hat{P}$ is the regular momentum of the measuring instrument. If the initial state of the quantum system and the measuring instrument is $|\psi\rangle \otimes|\phi\rangle$, Then the end state of the total system can be written as

$$
|\psi\rangle=\sum_{i} c_{i}\left|\psi_{i}\right\rangle \otimes\left|\phi_{i}\right\rangle
$$

If there is only the inverse effect, can the wave packet collapse and the quantum decoherence occur when a particle to be measured? We can find the answer in the logical analysis and the discussion of experiment result. There are problems related to this: What are the occurring conditions for the quantum states superposition? What are the conditions for the quantum superposition state to be destroyed? The understanding of these two problems by orthodox quantum mechanics scientists is rather vague. Saied to them, they also are vague word. In fact, they actually identified that the superposition of a microscopic particle with its own shadow is unconditional, and the resistibility of the quantum superposition state to the external influence is zero. Quantum mechanics often use this point of view to lack theoretical basis. Orthodox quantum mechanics scientists believe that the coupling between the measured particle and the instrument leads to the destruction of the quantum superposition state described by formula (9), so that the measured particles back to the classical state. Although these two kinds of knowledge (this one and "original state superposition of microscopic particles is unconditional and inevitable") lack theoretical basis in physics and only take the Hilbert space as the mathematical basis, but they are regarded as the golden laws and precious rules in quantum mechanics. So it is not rigorous, contrary to the spirit of science. Below we will discuss in more detail the coupling condition and the anti interference ability of quantum superposition state.

Both Equation (9) and Equation (11) are written by the assumption that the measured particles are entangled with the environment. The irrational concept that the state stack is not limited by the distance of the action is used. In fact, 
according to the description of the 6.2 section, as long as the single particle and measuring instrument of separated by a certain distance, or the field that environment effects on the investigated particle is weak to a certain extent, there is no reason to use Equation (9) and Equation (11).

If the anti interference ability of the quantum superposition state (the original, spontaneous superposition state) is zero, then, the quantum superposition state can only exist in the ideal environment with absolutely no interference. However, this kind of ideal environment does not exist in reality (any particle will be at least affected by neutrinos, gravitational fields and other cosmic noises, and bound electrons will be affected by the electromagnetic field). There are many kinds of indefinitely the fact that measurements (or observations) have not changed the quantum state of the observed object in reality. For example, the observation of diamond cannot make its $\mathrm{sp}^{3}$ hybrid state of the slightest change. The spin direction of the paired electrons in the diamond internal hybrid orbital is also difficult to be changed. To know, the orbital hybrid is also a quantum state superposition. Two homologous conjugated particles separated by $1.3 \mathrm{~km}$ (or infinity) are not independent individuals, which lacks the objective evidence that there is a logical connection between particles. It does not conform to the logic that interference-free measurement can also change the quantum state of the logic. How can an interference-free measurement (or an observation without deliberately changing the polarization direction of the photon and the direction of the electron spin) change the polarization direction of the photon and the spin direction of the electron? It is concluded that, if the anti interference ability of quantum superposition states is zero, and the quantum superposition state (the original, spontaneous superposition state) does not exist in reality. The concept of quantum state superposition and the concept of quantum decoherence (or wave packet collapse) need that quantum superposition state have a certain ability to resist interference (the anti interference ability of the quantum superposition state cannot be zero).

Lee Rozema in the quantum optics research group of the University of Toronto has designed a device for measuring object properties. The results of the study are published in September 7, 2012 in the Physical Review Letters [4].

In order to achieve this goal for measurement of interference as little as possible, it is needed to measure before the photon enters the instrument. But this process can also cause interference. In order to solve this problem, Rozema and his colleagues used a weak measurement technique; let the interference of the measured object very little. Before each photon is entered into the instrument, the researchers are weak to measure it, and then use the instrument to measure, finally, compare the two results. It is found that the interference caused by them so big unlike the deductions of Heisenberg principle. This finding is of disbenefit to the uncertainty principle and the Neumann quantum measurement standard model.

The results of the study by S. Kocsis, B. Braverman, S. Ravets, M. J. Stevens, R. P. Mirin, L. K. Shalm, and A. M. Steinberg in 2011 in the Science magazine the weak measurement introduced has directly proved that the interference fringes 
have not disappeared after the quantum decoherence [5]. Serge Haroche and David Wineland independently invented and developed the methods to measure individual particles in the case of keeping the quantum mechanical properties of individual particles. The particles are still in the potential well, but their quantum properties still seem to be not destroyed. This is subversion before people think that the view cannot be observed directly (to allow the particle to rest is to measure and is the interference of the instrument to the particles. The quantum properties still exist. This shows that the original spontaneous quantum superposition state is not destroyed in the measurement).

The above experimental results show that the anti disturbance ability of the quantum superposition state is not zero. That is not to show that once it is observed, the quantum superposition state will collapse and disappear. In particular, they do not show that superposition state collapsed will be also occurred when the consciousness of people want to observe act on the observed object. In other words, as long as the anti interference ability of the quantum superposition state is not zero, there is the measurement that Quantum Coherent States is not destroyed. There is this kind famous experiment in the experiments that has been done. The development trend and ultimate goal of weak measurement is interference free measurement (i.e. reverse measurement).

In order to be more intuitive, we list Table 1 to compare and analyze several different situations.

Instrument interference (impact) measured particle is showing that the instrument can change the state of the microscopic particles being measured. If there is no contact between the two systems, and the information transmits in one-way between the two systems, the role is also a one-way. If only the information emitted from the measured microscopic particle to the measuring

Table 1. Analysis of measurement results of information reverse transmission

\begin{tabular}{|c|c|c|c|}
\hline Case & $\begin{array}{l}\text { The type of signal of emitting } \\
\text { from the measured particle } \\
\text { to the instrument (observer). }\end{array}$ & $\begin{array}{l}\text { The effects of the observation and } \\
\text { no observation on a measured particle. }\end{array}$ & $\begin{array}{l}\text { The state superposition (coupling) } \\
\text { between the measured particles and } \\
\text { the instrument. Observed results. }\end{array}$ \\
\hline 1 & $\begin{array}{l}\text { Field signal (electric field, } \\
\text { magnetic field, } \\
\text { gravitational field) }\end{array}$ & $\begin{array}{l}\text { The same: Whether or not observation, } \\
\text { the measured particles are not affected (interfered) } \\
\text { by the observer (instrument). }\end{array}$ & $\begin{array}{l}\text { State superposition (coupling) is } \\
\text { very reluctantly. The measured } \\
\text { particle is undistorted. }\end{array}$ \\
\hline 2 & $\begin{array}{l}\text { Photons, neutrinos, } \\
\text { sound waves }\end{array}$ & $\begin{array}{l}\text { The same: Whether or not observation, } \\
\text { the measured particles are not affected (interfered) } \\
\text { by the observer (instrument). }\end{array}$ & $\begin{array}{l}\text { State superposition (coupling) is } \\
\text { very reluctantly. The measured } \\
\text { particle is undistorted. }\end{array}$ \\
\hline 3 & $\begin{array}{l}\text { objebt particle } \\
\text { (electron, neutron, } \\
\text { proton, ion, atom) }\end{array}$ & $\begin{array}{l}\text { As long as the signals are not bounced } \\
\text { back to the source of the launch, Whether } \\
\text { or not observation, the result is the same. }\end{array}$ & $\begin{array}{l}\text { Under the conditions of set, the } \\
\text { measured particle will not distort. State } \\
\text { superposition (coupling) is very reluctantly. }\end{array}$ \\
\hline 4 & $\begin{array}{l}\text { Measured particle directly } \\
\text { contact with instrument. }\end{array}$ & $\begin{array}{l}\text { No Difference: The observer (instrument) } \\
\text { has a reaction force, which has a serious } \\
\text { effect on the measured particle. }\end{array}$ & $\begin{array}{l}\text { May conform to the condition of state } \\
\text { superposition (or coupling), distortion. }\end{array}$ \\
\hline 5 & $\begin{array}{l}\text { Measured Particle is trapped } \\
\text { (or absorbed) by instrument }\end{array}$ & $\begin{array}{l}\text { Difference: the instrument (observer) } \\
\text { has a serious effect on the measured particle. }\end{array}$ & $\begin{array}{l}\text { May conform to the condition of state } \\
\text { superposition (or coupling), distortion. }\end{array}$ \\
\hline
\end{tabular}


instrument, the particle does not change the motion state of the whole instrument. Therefore, the processes (and/or results) that the instrument affects the particle and the particle is influence on apparatus are asymmetry (the damage of the superposition state and the coupling between the particle and the instrument caused by that the instrument affects the particle and the particle is influences on apparatus are asymmetric).

If the signal on each row in the table is transmitting in the opposite direction, the measured particle will be subject to interference by the instrument. This is the performance of the asymmetry of the above mentioned. In the case of first lines and second lines in Table 1, whether the target particle is observed, both the motion state of the target particle and the signal emitted by the target particle are not changed (Note: the target particles are the measured particles). The reason is that the parts of the inverse signal come into the visual organ of the observer when a person is observing the target particle; the signal is received by environment when no one is observing it. There is no difference between the two conditions (the inverse signal to be received by ones visual organ and the inverse signal to be not received by the environment) for the target particle.

If the first two cases in Table 1 can also cause the coupling between the measured particle and the instrument, the original superposition state of the measured particle is destroyed, so that the target particle should be coupled with the environment (because, the environment is receiving the information from the target particles when no one is measuring). There are two problems in this way: first, if the coupling between the measured particle and the environment is unconditional, no one can get the original superposition state of the microscopic particle described by Equation (9), under any circumstances (any so-called quantum properties cannot be observed)? Second, how far can the particles are coupled with the environmental matter? If you cannot satisfactorily solve these two problems, just believe that the first two cases in Table 1 cannot cause the coupling between the measured particle and the instrument, the original superposition state of the measured particle cannot be destroyed. If the ability of the microscopic particles to resist the external disturbance described by Equation (9) is not zero, we can change the "believe" to "firmly believe". In this case, there is a larger space for the realization of quantum inverse measurement.

\subsection{The Facts and Experimental Phenomena Which Are Not Supporting the Existing Interpretations of Quantum Mechanics}

Section 2 enumerates the fact that the existing quantum mechanics is not supported (it is to look at those facts from the different levels and angles). This section will give some more specific analysis of those facts and phenomena.

\subsubsection{The Root of the Confusion of the Explanation and Understanding of Quantum Mechanics Is Also That It Does Not Match the Experimental Facts}

For explaining and understanding quantum mechanics, the situation is chaotic. 
This indicates that there is a problem with the existing quantum mechanics explanation. This indicates that there is a problem with the existing interpretation of quantum mechanics. In order to make this research meaningful, it needs to exceed Einstein in theoretical depth and uniqueness. As long as we is in the existing experimental facts to find the material of refurbishment of quantum mechanics interpretation, It is easy to find that electron diffraction experiments, known as the lifeline of quantum mechanics, also present the exact facts that deny existing interpretation of quantum mechanics. The breakthrough point is to look at the whole experience of the electron beam in contact, and it is found that the quantum coherence of the electron beam is independent of the experience before it passing through the slit. This "irrelevance" does not support the concepts of wave packet collapse and quantum decoherence. For quantum entanglement experiments, the change of quantum state has not been verified by experiments (because a single observation of a particle's state does not verify a change in states). Since the state change has not been experimentally verified, the super-distance correlation between the entangled particles has not been experimentally verified. To the hypothesis of "quantum state change due to measurement" as the premise, the experiment conclusion that has been confirmed by experiment was obtained. There is a significant logic loophole in this process. The "irrelevance" in the above electron diffraction experiment and the "logical defect" in quantum entanglement experiment together determine that the existing interpretation system of quantum mechanics is incomplete, and the system cannot be supported by all experiments. The most annoying irrelevance and the biggest new logical loophole in quantum mechanics have been found by me. I am sure that, for similar studies, I have exceeded Einstein in theoretical depth and uniqueness.

In quantum mechanics, the quantum state is not a physical quantity, there are also measurement problems. Therefore, it is not surprising that there are the interpretation problem and other basic problems of quantum mechanics. Some people do not think there is interpretation problem, and some people adopt a mixture of some or several interpretations, or some kind of personal understanding. For the basic problems of quantum mechanics, in the general teaching and research rarely involved, only a small number of physicists concerned, and did not reach a consensus. These situations probably reflect that the basic problem of quantum mechanics has not yet been fully solved. Many physicists take pragmatism and only use quantum mechanics as a calculation rule. This behavior is only to avoid the problem without solving the problem, but cannot say that the problem does not exist.

Including the death of Einstein, there are many people questioned the basis of quantum mechanics [6]-[11]. Exposing and resolving problems in quantum mechanics can serve as a research goal. Einstein's accusations of quantum mechanics did not mention the key points. The refutation of others is less than Einstein. In order to make this research meaningful, it needs to exceed Einstein in theoretical depth and uniqueness. I also take to expose the basic problems of 
quantum mechanics as a research goal. The method I use is different from others (I analyzed every detail of the measured particles in the whole process of the experiment, and avoided to look at their partial performance during the experiment in isolation. An irreparable new logic flaw in quantum entanglement experiment is found).

Many people believe that the existing experimental facts support quantum mechanics without exception. However, this understanding is too arbitrary. On the one hand, experiments and facts that do not support the existing interpretation of quantum mechanics are present. On the other hand, so-called experiment support of quantum mechanics refers to all experiments support mathematical form system of quantum mechanics, rather than the existing interpretation system of quantum mechanics to be supported by all experiments. Moreover, the wrong understanding and explanation can also make the experiment that original does not support a theory support this theory. We can all find examples of these.

\subsubsection{The Retention and Play of the Diffraction Properties of the Moving Electrons in the Vacuum Are Independent of Its Previous Experience}

Quantum coherence (quantum parallelism, quantum entanglement, quantum cannot be cloned, the diffraction and interference of particles, etc.) is the characteristic of microscopic particles different from macroscopic objects. It is also the birthplace of quantum theory. Quantum decoherence is the disappearance of such quantum coherence. In the existing quantum mechanics explanation, the description for measuring instruments influence of the measured system is mainly that the environment led to the collapse of the wave packet. The basic idea that the environment leads to decoherence is: Any physical system will not be completely isolated from the environment, and the interaction between the system and environment will lead to the entanglement of the system and the environment (some call it association, others call it superposition). The prerequisite for the environment to lead to decoherence is that measurements can cause waves-packet to collapse. Simply put, "as long as the measurement, quantum coherence disappears." The following discussion shows that there is a serious contradiction in the view of quantum mechanics. The most famous double-slit diffraction experiment by electron does not always support the existing interpretation of quantum mechanics.

In fact, the electron diffractometer and the power supply are in a current loop with generators and electrical appliances (we only consider the current loop consisting of one generator and one electron diffractometer). When the diffractometer is in operation, the electrons that form the current move directionally in this loop and undergo diffraction when passing through the slit. In the generator and the wire, those electrons that undergo the diffraction are affected by the electromagnetic field. In particular, electrons are subjected to strong electric fields (more strongly than in the wire) on the cathodes in the circuit. The electron beam leaving the cathode is generally subject to the collimation of the 
magnetic field. The intensity of these effect are no less than the intensity of the instrument acting on the electrons in general measurements (for example, using the cloud chamber and the spark chamber to measure incident electrons). According to the existing measurement view of quantum mechanics, the electrons in the generator and on the cathode should be in a quantum deco- herence state. But in fact, they are not in a state of decoherence, and diffraction can still occur in the electron beam. The 5000 electrons flowing through the generator are numbered by us. In these 5000 decoherence electrons, some of them will flow into the electron diffraction instrument and participate in electron diffraction. Thus, there is a problem that decoherence electrons recover quantum coherence. After the diffraction, the numbered electrons return to the wires of the closed circuit, and the quantum decoherence occurs once again as the role of the magnetic field in the generator and the role of the strong electric field on the cathode. They continue to flow and can take the next round of diffraction (coherence is restored). As long as the system works continuously, the above cycle can occur countless times. Each cycle requires: $<1>$ "quantum decoherence process and the superposition process of quantum state to be reversible", or, <2> "quantum coherence of moving electron in vacuum has nothing to do with past experience of these electrons" (referred to as this fact is "irrelevance" hereinafter), or $\langle 3\rangle$ the wave packet collapse process and the quantum decoherence process do not exist or do not occur. The assertion does not hold water that measured system and the measuring instrument are inseparable (the recovery process of quantum coherence just is the process that measured system get rid of the instrument interference).

If the electrons always have diffractive properties in all experiences before passing through the slit, the argument that "any measurement will inevitably lead to wave packet collapse and quantum decoherence" is not correct, and the pure and objective state of the particles can be observed (quantum inverse measurements can be implemented). The final conclusion is that the coherence of moving electron in vacuum is not related to its past experience". In this case, many quantum entanglement experiments must be rewritten. Both the action of the strong electric field on the electron beam and the effect of the magnetic field used for collimation on the electron beam are in line with the measurements defined in this paper. These measurements are the local measurements which do not lead to quantum decoherence and wave collapse. The experiment that electrons subject double layer lattice and undergo secondary diffraction directly proves that the measurement for the electrons passing though a double-layer slit cannot cause the wave packet collapse. The reason is that only the wave packet collapse did not occur when the electrons pass through the first slit, the electrons continuously advancing can undergo second diffraction. In view of the importance of the electron secondary diffraction experiment, the experiment will be repeated and analyzed below.

The "irrelevance" mentioned above is one of the most important conclusions of this paper. It is based on experimental facts. Please the readers whom try to 
deny this article first denies this irrelevance. I do not know how the authors and the pious readers of these articles [12] [13] [14] [15] look at this "irrelevance".

As mentioned above, in an electron diffractometer, the diffracted electrons are transmitted from the cathode plate rather than newly generated (they are affected by the magnetic field in the generator, obstructed by the metal atoms and the electrons in the wire, affected by the strong electric field outside the cathode, and affected by electromagnetic lenses). Diffraction can also occur when electrons coming out of the cyclotron or linear accelerator pass through the slit. This indicates that the magnetic field, the electric field, and the internal environment of the conductor cannot destroy the quantum coherence of the flowing electrons (or quantum coherence can be restored under certain conditions). The electrons can also undergo secondary diffraction when passing through the appropriate crystals. This indicates that the slit leading to the first diffraction as a measuring instrument does not lead to the electrons to produce quantum decoherence. The electron beam in the electron microscope is collimated by an electron lens, and the electron lens does not cause the quantum properties of the electron beam to vanish. The stationary ions trapped in the microcavity can also maintain the quantum coherence of quantum entanglement. Considering the mechanism of the secondary diffraction of electrons, Electronic double slit diffraction experiments show that there is only the state superposition between the measured electrons, rather than between the instrument and the incident electrons. The fact that the optical signal is difficult to be distorted by the long distance fiber does not support the inevitability of the association (superposition) and the collapse of the wave packet between the instrument and the measured particle. Numerous facts show that the quantum coherence of free electrons in vacuum is independent of the source of electrons (i.e., the electrons with the same velocity in the vacuum are not distinguishable). Popularly, "no matter what the sources of the electrons are, the electrons that move in the vacuum have quantum coherence, which can be diffracted." This conclusion shows that "wave packet collapse", "the quantum state superposition between measured system and instrument (environment)" and "the inseparable between measured system and instrument" and other concepts are not correct.

The electron beam can produce diffraction. This quantum coherence is independent of the source of the electron beam (independent of the previous experience of electrons). Is quantum coherence formed (restored) at the moment the electrons leave the source? A variety of different quantum coherence should not be independent of each other. If the electron diffraction characteristics can be restored, the quantum entanglement properties can also be recovered. The quantum entanglement of twin electrons is also independent of the previous experience of homologous electrons. For example, the $4 s^{2}$ electrons of a calcium atom are emitted and then reflected back to the $4 s$ sublayer, and the entanglement between the $4 s^{2}$ electrons can be restored. If the quantum coherence cannot be recovered, decoherence process is irreversible, that is, quantum coherence does not exist or can withstand considerable intensity interference. In this case, 
the experiments using cloud chamber and the spark chamber to capture the movement trace of electrons deny the principle of uncertainty (especially the assertion that "it is also uncertain when there is no measurement and no interference"). If the decoherence process is reversible, the physical quantity has many different eigenvalues and the eigenvalue is random is denied, also denied the existence of the process of wave-packet collapse (no need to the concept of wavepacket collapse).

Although the moving particles in vacuum are affected by the electric field and the magnetic field still maintain the diffraction properties, they still retain diffraction characteristics. This fact indicates that the anti-jamming capability of the quantum coherence of the particles is not zero. We can find the measurement methods that there is any interference or interference can be ignored, and the pure objective state (or the purely objective state) of the particles can be observed. In another way, "the diffraction characteristics (one of the quantum coherence) of the moving electrons are independent of the origin of the moving electrons". There are three possible reasons for this fact: first, the wave packet collapse process does not exist; Second, the wave packet collapse process (or quantum decoherence process) is reversible; Third, the diffraction of electrons and other object particles is not directly caused by the object particles, but the side effects of particle movement. These possibilities are detrimental to the existing interpretation system of quantum mechanics.

In the above-mentioned irrelevance performance, the various experiences of electrons before diffraction are that they undergo a variety of different measurements. These measurements do not destroy the coherence of electrons, which do not result in wave packet collapse and quantum decoherence. This is the measurement that the interference can be ignored underwent by electrons (this is what the author called reverse measurements). Thus, enumerations of these examples are experimental evidence that quantum inverse measurements can be achieved.

\subsubsection{The Concept of Quantum Entanglement Lacks Solid Theoretical Basis and Experimental Basis}

At the beginning of the concept of quantum entanglement, for the instantaneous quantum entanglement experiment, there is the interpretation that just the birth of the twin particles is a clear pigeon pair. The existing quantum mechanics (specifically the year of Bohr) denies this possibility with the principle of uncertainty (he use is the assertion that conjugate physical quantities are also uncertain in the absence of measurements or interference). However, the uncertainty principle does not specify the spin state also has uncertainty. We know that some quantum states are not physical quantity, only the certain physical quantity to meet the principle of uncertainty. Weinberg said that we cannot find the theoretical source of the probability of quantum mechanics (S. Weinberg, 2017). In this way, the theoretical source of the assertion that conjugate physical quantities are also uncertain in the absence of any measurement or interference is also not found. Logically, this assertion cannot be experimentally verified. If combine 
the discussion of this paragraph with the discussion of the previous paragraph, we can obtain the conclusion that Bohr's method is very far-fetched.

In theory, two fermions are not allowed to have exactly the same quantum states. In fact, two $1 s$ electrons (also two fermions) are identical particles. Both need them to be two states, and must admit that they are indistinguishable. This is contradictory. In order to solve this contradiction, it is assumed that there is an entangled state. In other words, $1 s^{2}$ electrons are admitted to have a difference between spin up and spin down (it is the requirements of Pauli incompatibility principle). However, identical fermions require that they not be distinguished. In order to solve this contradiction, it is assumed that there is an entangled state. It can be seen that the theoretical basis for the existence of quantum entangled states is weak.

The process of the state evolution of wave-packet collapse caused by measurement cannot be described by the Schrodinger equation. The fact that the electron beam can undergo secondary diffraction shows that the first measurement did not cause the wave-packet collapse (quantum decoherence). The diffraction characteristic of the electron beam is independent of its previous experience, but also has nothing to do with the role of the electric field. These three facts together show that the assumption that "measurements would necessarily lead to wave-packet collapse (quantum decoherence)" is not true. It is also shown that continuous measurements cannot led to the particles to remain always in quantum decoherence.

The theoretical basis of the concept of quantum entanglement is the contradiction between "incompatible principle" and "all the same fermions to be indistinguishable", and the principle of uncertainty. It is this pair of the contradictions and the principle led to a kind of helpless choice, and is a hypothesis. There are logic loops and the problems of cyclic argumentation in quantum entanglement experiments. The concept of quantum entanglement has neither a solid experimental basis nor a solid theoretical basis.

\subsubsection{Quantum Entanglement Experiments Cannot Confirm the Existence of Quantum Entanglement (Super-Distance Association)}

Does the quantum entanglement experiment support the existing interpretation system of quantum mechanics? As long as careful and careful analysis, it is not difficult to find a definite answer. Now, quantum entanglement experiments are divided into two categories: The first category is the effect test of instantaneous entanglement; the second category is the discovery and verification of continuous entanglement. The experiments for the effect test of instantaneous entanglement have a very large logic vulnerability - the change in the state of one of the twin particles has not been experimentally verified but is inferred from a certain idea. If you want to use experimental methods to verify the change of a particle's state, you must observe the particle two times before and after. However, the existing instantaneous quantum entanglement experiments only make one measurement (observation) of the state of one of the twin particles, and the change of the quantum state is the result of theoretical speculation rather than 
being found (validated) by measurement. This kind of experiment has no way to exclude "the twin particles are definite pigeon pair at the time of their birth." If the twin particles are the definite pigeon pair at first, it cannot be said that this experiment proves the instantaneous entanglement of the twin particles (how can there be the verification of "paranormal association of state change" when the change of state is not verified). In other words, for instant quantum entanglement experiments, Physicists do not use experimental methods to verify the existence of quantum entanglement, but suppose that quantum entanglementstate exists before the experimental operation. It is from concept to argue that the experimental operations destroy (change) the already existing quantum entanglement-state". The earlier "irrelevance" conclusion also shows that even if the measurement does not necessarily lead to coherence disappear. If we consider the aforementioned "irrelevance" and "state change to be not verified" at the same time, the experimental conclusion "detection of the super-associated quantum entanglement" even more do not fly.

The twin photon entities are independent (they can be separated and can be separated very far). But their states cannot be independent. This is a freak in itself (the equivalent of the bodies of the pigeon pair are independent, but their reproductive organs and chromosomes mixed together), and it is imagined (which is not derived from the wave function, nor is it found in the experiment. The principle of indiscernibility of homologous fermions is not effective for twin photons). Some people may say that this is the characteristics of micro-particles. However, even for micro-particles, that they have a strange entangled state, but also must find the theoretical basis and experimental basis. As the current method of verification of quantum entanglement is not reliable, therefore, the so-called measurement of the existence of entanglement, are the experimenter self-talk.

There are also significant logical problems in continuous quantum entanglement experiments-it is observed by the experiment that Schrödinger cats change alternately between the dead cat state and the live cat state, rather than the superimposed state of death-live cat. Theoretically, the persistent entanglement between the twin particles A and B should be the continuous superposition (or mixing) of the state 1 of $\mathrm{A}$ and the state 2 of $\mathrm{B}$, rather than the alternating conversion between the state 1 and state 2 . In this case, the quantum entanglement is considered to be experimentally verified, but in fact, it is not logical (we need to change the original definition of quantum entanglement and the content of the principle of state superposition). It is observed that "the twin particles are alternately converted between state 1 and state 2 ". It is observed that the two particles are the pigeon pair. It's just the sex instability of the twins (the dragon changes into a phoenix, at the same time, the phoenix changes into a dragon. Although this change does not stop, but the dragon body and the phoenix body is clear and independent. It has been measured that this alternating change is sustainable for $50 \mathrm{~ns}$. Note: the dragon represents the male, and the phoenix represents the female). This indicates that the "superluminal correlation between 
entangled particles" has not been experimentally confirmed. In other words, the continuous entanglement experiment proves that the twin particles are pigeon pair before the quantum state is destroyed by experimental operation, and denies the existing conclusions of the instantaneous quantum entanglement experiment. If we believe that quantum entanglement is a mixture of dragon and phoenix (no independent dragon body and phoenix body), it is denied the experimental conclusion of continuous quantum entanglement. It can be seen that the conclusions of the existing continuous quantum entanglement and instantaneous quantum entanglement are mutually negative. The quantum scientists cannot explicitly answer the question which is the quantum entanglement state a quantum state uncertainty or quantum state instability?

There are two processes of quantum state evolution, one is, before measurement, the evolution to be described by the Schrodinger equation, which is reversible and deterministic; the other is the collapse caused by measurement, which is irreversible and random. Why is it irreversible? Since the quantum state before the measurement can collapse to one of several states, the state before the collapse cannot be determined according to the state after the collapse. This change is not harmonious with the evolution of the Schrodinger equation, and is regarded as a basic assumption of quantum mechanics. That is, the entangled state (the superposition state of the twin particles) is assumed, and it is also assumed that the superposition state of the measured twisted twin particles is assumed. In this way, in the logical order of the measurement process of instantaneous quantum entanglement, it is assumed that the entanglement phenomenon exists, and finally the conclusion is that the entanglement phenomenon exists. This is a very obvious logical cycle that is the biggest logical loophole about quantum entanglement experiments. "Both twin-electron entities are independent, but their state cannot be independent" is a freak (i.e., the premise in the logic sequence of the experiment of instantaneous quantum entanglement is not common). It can clearly be seen, the experiment conclusion of the instantaneous quantum-entanglement is neither a logical conclusion nor the conclusion to be validated by experiment. Moreover, other logic loopholes of the experiments to validate Bell's inequality are not all blocked.

\subsubsection{The Significance and Follow-Up Work of Section}

The so-called experiments have confirmed the correctness of quantum mechanics, mainly refers to the experiment of quantum entanglement. However, as long as the twin particles at the time of birth are pigeon pair, The Bell's inequality criterion is invalid (not working). In particular, the fact that the diffraction properties of electrons are independent of the previous experience of the electron beam is very detrimental to the existing quantum concepts.

The above is mainly about the issue of Copenhagen's explanation. Other explanations are equally problematic. "Multi-world theory" regards the quantum state itself as an objective nature, and there is no collapse, and all possibilities are contained in the huge quantum state of the whole world. This interpretation is burdened with a heavy metaphysical burden. Are there any links between different 
worlds? If there is physical contact, is that not a world? If there is no physical connection, how do different worlds coexist in a "super-world"? What is the world of this "super-world"?

I also agree with the Nobel laureate Weinberg said, it seems that each interpretation has its own problems, and also agree with Professor Yu Shi's additional comments, the problems of variety interpretations may be essentially different performance of the same problem (Yu Shi, 2017). I think their common ground is inseparable from the ghost. Copenhagen interpretation takes particle as a ghost, and the hidden parameter interpretation takes interaction as a ghost, and the multi-world theory takes the universe as a ghost.

Some people say that the impact of the environment will destroy the quantum entanglement, and some people say that laser irradiation of silicon carbide can create quantum entanglement. So, the impact of the environment in the end is to destroy the entangled state or create entangled state? At present, the method of verifying quantum entanglement is extremely unreliable. The reason for the existence of the accepted quantum entanglement is mainly that the state of homologous twin particles is also uncertain when they are not measured. However, logically, this assertion cannot be verified by any experiment. Can we say that quantum entanglement has been rigorously verified? It is fact that the coherence of microscopic particles has nothing to do with its past experience. This experimental fact has a heavy blow to the existing interpretation system of quantum mechanics. If "as long as the impacted by the environment, the wave packet will collapse", there will be no any quantum wave package of collapse-free in the nature. If "as long as the impacted by the environment, entangled state will be destroyed", there is no quantum entangled state in nature. The reason is that the observed particles cannot be isolated. It is obvious that the experimental conclusions about quantum entanglement cannot stand close scrutiny.

In the above case, if there is an interpretation system of quantum mechanics for localized realism, which interpretation system would you choose?

\section{Experiments with Conforming Quantum Inverse Measurement Conditions}

The purpose and function of quantum measurement is to obtain information about the micro world. As long as the information is transmitted from the measured object, the purpose of quantum measurement can be achieved, and it is not necessary to send information to the measured object. In this way, there must be a class of measurements that do not interfere with the state of the quantum system in the measurement process and can continuously read certain observable quantity. Existing quantum nondestructive measurement is limited to projective measurement, and it belongs to the category of quantum inverse measurement, but it is not the core of quantum inverse measurement. Any measurement that does not destroy the quantum superposition state or does not lead to the collapse of the wave packet is a protective measurement (It can be local or all. It is not necessary to meet the conditions of quantum Zeno effect), and 
meet the conditions of generalized quantum inverse measurement. Measurements outside the projection measurement of the Bonn are likely to be consistent with the conditions of quantum inverse measurements. See sections 2 and 3 for details.

In fact, in the existing concept of quantum mechanics, the quantum state is not real movement speed, movement direction, and fluctuation mode and so on, but the particle spin, polarization, superposition and wave packet width. The superposition of the state is the integration (fusing) of nothingness. The idea of identical particles indicates that it is meaningless to measure and distinguish the spin and spin direction of the electron. For photons, the operation to measure the direction of polarization does not change the polarization direction of the photon. Using micro particle structure, Section 6.2 shows that the general measurement is mainly to change the motion state and energy of the particle rather than change the others.

\subsection{The Measurement Which Does Not Destroy Quantum Superposition State}

French scientist Serge Haroche and the United States scientist David Wineland won the 2012 Nobel Prize in physics. The reason is "for the discovery of measurement and manipulation of individual quantum system by experimental method, and realized "imprisoned and manipulation of the small number of atoms or ions system, which in the previous, it is considered to not be achieved".

T. Hanesch, D. Prichard, Cohen-Tannoudji, W. Phillips, C. Wieman, E. Cornell, W. Ketterle and others have done the work of cooling and imprisoned quantum. They found that: the same Penning ion in a Paul trap than in a lot of honest can stay in the center of the trap motionless. The same ion in a Paul trap is many honest than in a Penning trap, and can stay in the trap motionless. A string of the same ion can be imprisoned in the center of the four pillars and suspended into a line, at the same time, electrostatic repulsive force make them associated each other. The German Rampe team found that the momentum perturbation of the mass center of the cooling atom can be reduced to a negligible degree.

That is to say, some of these Nobel laureates allow individual particles at rest in the trap. In this case, the position and momentum of the particles can be measured simultaneously accurate, and the kinetic energy is zero. Imprisoned ion may be smaller proton. The still particles are full of particles (the waves have collapsed). It is clear that in these experiments, the packet collapse occurred, but quantum decoherence did not occur.

In the Leibfried's experiment introduced by Ref. [16], the researchers fixed beryllium ions in electromagnetic field wells at intervals of several micrometers and then cooled the beryllium ions to nearly absolute zero by laser and manipulated these ions in three steps exercise. The laser is then used to cool the beryllium ions to near absolute zero and manipulate these ions in three steps. In order to allow as many particles as possible to achieve "Schrödinger cat" state for as long as possible, Researchers on the one hand to improve the cooling efficiency 
of the laser, on the other hand, as much as possible to absorb electromagnetic field wells to send the heat of ion vibration. In the end, they caused 6 beryllium ions to spin in both clockwise and counterclockwise directions simultaneously in 50 microseconds, the same amount of superposition entanglement of two opposite quantum states is realized, that is, the Schrodinger cat state. Researchers at the University of Innsbruck, Austria, also reported in the same issue of Nature that they achieved a "Schrodinger cat" state in 8-ion systems, but maintained for a shorter time [17].

The entanglement in these reports is defined by the experimenter. They are not necessarily a true quantum entangled state. The reason is that humans do not know the essence of the real quantum entanglement process, the state of entanglement in the end is what we do not know. The reason is that humans do not yet know the essence of the real quantum entanglement process, do not know what the entangled state is like. If an ion is in a clockwise spin and counterclockwise spin state, the whole is not spin and is difficult to be perceived. Most importantly, beryllium ions are not distinguishable. A beryllium ion of clockwise rotation rotating 180 degrees is a beryllium ion of counterclockwise rotation. Beryllium ions continue to flip on the performance of that look, not Schrodinger's cat state.

It is meaningless to talk about the static of non-localized things. Particles that can remain stationary must not be delocalized.

\subsection{The Measurement That Has Not Cause the Collapse of the Wave Packet}

There is an experimental phenomenon called secondary electron diffraction. It is high-speed electrons passing through the first slit and generating diffraction. After that, the electrons pass through the second slit and generating diffraction again. If the collapse of wave packet is reversible, the probability explanation is destroyed. Therefore, the direct conclusions of the experiment are: continuous slit measurements did not result in wave packet collapse (or the state-superposetion between the instrument and the measured particle); second, there is a definite motion path when an electron from the first slit to the second slit (the electron is not scattered waves). The experimental results show that, either "wave packet collapse does not exist" or "the process of wave-packet collapse is reversible." As long as the wave-package collapse does not exist, cloud chamber and other instruments to capture the state of the particles can only be a pure-objective state of the particle. The influence of the slit on the incident electron is much greater than that of the cloud chamber on the incident electrons. If the slit does not cause the wave packet of the incident electrons to collapse (or the state is superimposed), the vapor fraction in the cloud chamber does not cause the wave packet of the incident electrons to collapse. The secondary diffraction experiment of electrons to be combined with experiments such as cloud chamber and other equipment to capture charged particles can form a complete the experimental evidence of denial of the existing interpretation about uncertainty relation. 
The experimental combination is also strong experimental evidence that the uncertainty paradox exists (Runsheng $\mathrm{Tu}, 2017$ ).

The diffraction experiments of electrons and photons can form a diffraction pattern that only waves can cause. After the first diffraction, the electrons can diffract again (this is the second diffraction of the electrons). This proves conclusively (as evidenced by experimental methods) that the slit (its width is about 1 angstrom, and is also an instrument) does not cause the measured particle to collapse. It is generally accepted that wave-collapsing occurs at the moment of particle contact with the screen. If you do not explain this phenomenon as "wave packet inflation"-the reverse process of wave packet collapse, but use the Copenhagen interpretation, it is necessary to recognize that the local process the microscopic particles pass through the slit conforms to the condition of quantum inverse measurement (just the process of particle arrival on the screen does not conform to the quantum inverse measurement condition). It is believed that measuring the polarization state of a photon with a polarizer inevitably destroys the superposition state of the twin photon by Copenhagen interpretation. However, the width of the gap of the fence column in a polarizer can reach tens of millimeters. A slit whose wide is $10^{-8} \mathrm{~cm}$ does not lead to the disappearance of quantum properties, and the barrier gap of a few tens of millimeters wide can lead to the disappearance of quantum properties? The success of the electron diffraction experiment shows that the strong electric field of the emitted electrons does not make the electron wave packet collapse. In addition, the electron beam collimated and focused by the electromagnetic field can still exhibit volatility in the electron microscope. The facts listed above show that the quantum properties of the particles may not be lost even if the instrument exerts an influence on the particles, at least partly in accordance with the conditions of quantum inverse measurements. Thus, the conclusion that the measurement of entangled photon polarization will lead to collapse of the wave packet is not reliable. If there are measurements that do not destroy the quantum state (which does not cause the collapse of the wave packet), the entire quantum mechanics interpretation system suffers a considerable impact (in fact, orthodox quantum mechanical interpretation and measurement concept was subverted).

An electron coming out of a cyclotron or a linear accelerator is still able to exhibit fluctuations and diffraction. Once again this indicates that the wave packet to collapse is not caused by the effect of the electromagnetic field on the electron. The process of accelerating electrons by electromagnetic fields is in accordance with the quantum inverse measurement conditions.

Nuclear decay is also one of the characteristics of microscopic particles. However, nuclear decay has nothing to do with all the conditions of the outside world, and it has nothing to do with whether to observe it. This situation is unquestionably consistent with quantum inverse measurement conditions.

\subsection{The Measurement Which Has Only Inverse Influence, or the Measurement That Positive Effects Are Weak and Negligible}

Based on the idea of direct observation of the robustness and the physical 
quantity, the Li Chuanfeng research group realized two kinds of quantitative measurement methods of the quantum coherence of the photon polarization. It is proved that the anti-jamming ability of the relevant quantum system is not zero [18].

The observation that the superposition state of Schrödinger's death-live-cat was observed is that it has been observed that the quantum entanglement lasts for a while by continuous observation. This observation during the continuous observation process does not destroy the quantum state, does not lead to wave packet collapse and the disappearance of state superposition. Observations (measurements) during this period accord with quantum inverse measurement conditions, it also meet the conditions of quantum protective measurements. This example shows that the anti-interference ability of the quantum state is not zero, as long as the interference intensity is less than the anti-jamming ability, the interference will not work, and the wave packet collapse will not occur.

When thinking about the question of whether the experiment of quantum inverse measurement can be realized, the first thing we think of is, in the experiments that have been done, whether there is the experiment to meet the conditions of quantum inverse measurement. The propagation of particles in the cloud chamber or the spark chamber is very similar to the propagation of photons in the fiber. The optical fiber does not distort the optical signal, and the cloud chamber and the spark chamber cannot distort the incident particles. The use of cloud chamber and spark chamber to capture the moving track of the micro particle, it is mainly completed by emitting the electric field signal form the measured particles to the measuring instruments. Instruments have almost no interference signals to the measured particles. The effect of the instrument on the measured particle is smaller and even order or very symmetrical, does not affect the objectivity of the measurement results. In addition, this measurement is a continuous measurement, the time interval between the two measurements is zero, and less than Zeno time, judgment according to quantum Zeno effect, the original state of the system can be observed. This kind of experiment is in full compliance with the conditions of quantum inverse measurement. Explain the motion of charged particles in the chamber by using existing measurement view originated from von Neumann's theory, there is a sharp contradiction (see next paragraph). Using QIMT to explain this kind of experiment can overcome this contradiction.

The proof derived by Neumann is wrong that the implicit function theory is unable to give the unique solution to the observations. This error was first discovered by David Bohm of a very famous scientist. Later, we all realized that Neumann's mistake.

As we all know, the thickness of the cloud chamber must be less than the penetration of the incident particles. The working principle of the chamber to capture the charged particle tracks is, the electric field of the target particle passing the "supersaturated steam" at high speed leads to ionization of nearby vapor molecules, the secondary electrons produced by ionization also lead to 
farther away from the vapor molecules to produce secondary-secondary electrons. This process occurs in the way to be similar to cascade shower and affects a larger range of steam molecules. The measured particle (target electron) does not reach the edge of the cloud track (the electrons reaching the edge are generated by secondary ionization). The measured electron (target electron) is still moving forward at high speed. In a word, in this range, only the measured particle affects the instrument, the instrument has little effect on the measured electron, and the superimposed state of the measured particle with its own shadow does not exist. The ionized molecule becomes the center of vapor condensation due to charge. That is, the high-energy particles injected into the cloud chamber can cause near-molecular ionization, so that the ionization process can be transmitted many times, every ion formed by ionization is the condensation center of steam. Since the steam is supersaturated, the agglomeration can be sustained and produce minute droplets. As a result, a sufficiently thick fog-belt appeared on the path which the particles passed. Under appropriate lighting conditions, you can see or shoot the past trajectories of the target particle. In the cross section of the cloud line (belt), the target particle is located at the regression center of the cloud point. The accuracy moving orbit of a target particle is the $3 \mathrm{D}$ regression curve of the drop center coordinates in the cloud track.

If it is considered that the $3 \mathrm{D}$ regression curve is not an accurate movement route of the charged particles at high-speed, it must be admitted that uncharged particles can also leave traces in the cloud chamber. If there is no collision, motion of a high-speed particle in the chamber is moving in the vacuum with a weak field. Taking into account the important "irrelevance" mentioned earlier", the measurement using the instruments such as cloud chambers and spark chambers is the continuous measurement without breaking the quantum coherence. It supports the theory of quantum inverse measurement and rejects the principle of uncertainty. The core of the working principle of the cloud chamber is the cohesion induced field. The center of the small droplets in the cloud trail is the secondary (or secondary-secondary) charge center (the seat of secondary field-source), and the 3D regression curve is the place passed by the center incident charge (the original field source), is the starting point to be similar to cascade showers. The original field source did not reach the edge of the track. If the measured charged particles will reach the edge of the cloud track, on the one hand, it does not comply with the principle of the chamber, on the other hand, it does not comply with the facts that the track is extremely regular (if the measured particles can reach the edge of the track, the cloud tracks cannot be so regular, and the $3 \mathrm{D}$ regression curve will not be so smooth). It is generally believed that the $3 \mathrm{D}$ regression curve is the classical trajectory of the particle. However, its positional accuracy can reach the atomic scale. This also indicates that the position and momentum are measured continuously and accurately enough, and the uncertainty relation does not hold true in the classical mechanics field. Uncertainty relations are also not true in the field of quantum mechanics.

Orthodox statistical interpretation also conflicts with the meaning of the $3 \mathrm{D}$ 
regression curve of the drop center coordinates in the mist track decided by the working principle of cloud chamber. As everyone knows, the thickness of the cloud chamber must be less than the penetrating power of the incident electrons. The working principle of the chamber to capture the charged particle tracks is that the electric field of the target particles passing through the "over saturated steam" at high speed leads to the ionization of the nearby vapor molecules, the secondary electrons produced by ionization are also like the charged target particles, which result in the secondary-secondary ionization of the further vapor molecules. This process occurs in the form of a shower and affects a larger range of vapor molecules. The measured particles did not reach the edge of the cloud track (the electrons of reaching the edge are generated by the secondary ionization). Measured electrons are still moving forward at high speed. In a word, in this context, only the measured particle impact instrument. The influence of the instrument on the measured electrons is small, and the superposition state of the measured particle and its own shadow does not exist. The ionized molecule becomes the center of vapor condensation due to charge. That is, the ions at all levels caused (induced) by the high energetic particles incoming the chamber as the source can become the condensation center of the over saturated steam, around these ion centers will produce tiny droplets, Thus, a sufficiently thick fog band appears on the path of the target particle. Under the proper lighting, we will be able to see or to shoot the past motion track of the particle. At the edge of the cloud track, obviously not the target particles arrive there, but a lot of secondary effects to have been caused by the target particles appear there. On the cross section of the cloud belt, the position of the target particle is at the regression center of the cloud point. The moving orbit of the target particle is the 3D regression curve of cloud fog band.

It does not conform to the facts that each droplet in the cloud fog band formed by the discharge and condensation of vapor molecule caused by the measured particles to visit there (it does not comply with the working principle of the chamber). It is the orthodox interpretation that the measured charged particles first superimpose with their own shadow, then coupling with the vapor molecules (also a superposition), such double superposition state leads to the formation of the fog band. This explanation requires that the cohesion center of each droplet is caused by the measured particle to visit there. This requires not only the super speed of light, but also, lack of mechanisms that the measured particles return the 3D regression center. In addition, this orthodox interpretation and the interpretation that the measured particle in a cloud chamber has returned to the classical state are contradictory. In the year of this explanation is to meet the principle of uncertainty and put it out of the facts. This explanation has been widely accepted (acceptance of the uncertainty principle must accept the interpretation of disregard of the facts). You know, even if the droplet track in the chamber is a superposition state space, the gravity center of the measured particle is also moving along the $3 \mathrm{D}$ regression curve of the droplet center coordinates in the track. 
In a cloud chamber, for high speed electronics, the distance between vapor molecules is great. The penetration ability of high speed particles (such as electron) is very strong. The probability of collision (in particular, the collision that can change the path) between target particles and neutral molecules is very low. Neutral vapor molecules have little effect on the high speed particles passed through (almost no influence, and the influence is less than the resistance). From the Rutherford's experiment of the detection of the atomic structure we can see, the effect of electrons in atoms and molecules on the high speed particles is very small, As long as there is no collision to the atomic nucleus, high-speed particles will not change the movement route. The electric field of the incident particles affects the environment (instrument) when a particle is captured by a spark chamber, and the environment (instrument) has little effect on the incident particle (the design idea of the spark chamber is that the motion path of the incident particles cannot be changed because of the influence of the instrument). Since the anti interference ability of the quantum coherent state is not zero, we only consider the effective measurement results that the impact strength is lower than the anti interference ability. For the experimental results using cloud chamber to capture the track of a charged particle, quantum decoherence interpretation is in contradiction to the interpretation that the measured particles have been visited every condensation center. The orthodox interpretation of the experimental results using spark chamber to capture the track of a charged particle also has the above contradiction. In order to overcome the contradiction, and considering the working principle of the chamber, we can recognize that the effective experimental results using cloud chamber trapping of charged particles track accord with quantum inverse measurement conditions. The motion track of micro particles (the 3D regression curve) obtained by measuring is the intrinsic state (undistorted state) of the particle. They are not due to be measured and returned to the state of the classical. To capture the same particle, the same track can be obtained by using spark chamber, cloud chamber and bubble chamber. This fact has proved that, in a short distance, effects of the equipment to capture particle track on the incident particles is negligible. The subjective intervention that the measured particles to be effected by the consciousness about an observer wants to measure the microscopic particles lack scientific basis.

When a high-speed particle passes though the chamber, the principle of formation of cloud track is of field induced aggregation. We only discuss the process before the collision of the incident particle with the vapor molecule. When the particle passes through the chamber, the electric field signal affects the steam molecule in the instrument, and the steam molecule has no effect on the incident particle. This fully conforms to the conditions of quantum inverse measurements. In the spark chamber, the electric field of the incident particle causes the space between the strings of the instrument to be broken down and discharged to produce a spark. The electric field between the strings is very weak and has little influence on the incident particles (much weaker than the electric field needed to make a electron beam). In the secondary diffraction experiments 
of electrons, the effect of the slit on the electrons being measured did not result in quantum decoherence and wave packet collapse. High-speed electrons penetrating into the cloud chamber are also likely to have no occurrence of wave packet collapse and quantum decoherence. The 3D regression curve of the center coordinate of the droplet in the cloud chamber is most likely to be an accurate movement route of the measured particle. The thickness of the cloud track is the reflection of the effective distance that the electric field of the incident particle can affect (neutral particles cannot form a cloud track is proof). If the incident particles are considered to reach the edge of the cloud track, superluminal motion is required and the segmented $3 \mathrm{D}$ regression curve is not a straight or smooth curve.

For the use of the measuring chamber, people always avoid talking about the significance of the 3D regression curve. Both the accuracy of the 3D regression curve and the characteristics of the motion path of the particles in the second half can be predicted according to the first half of the $3 \mathrm{D}$ regression curve are the powerful materials that deny the principle of uncertainty.

In summary, measurement of nuclear decay processes, Leibfried's experiment, the localization of electron diffraction experiments, the local of electron microscope, using cloud chamber and bubble chamber, spark chamber etc. to capture micro particle track, the effect of measuring instruments on microscopic particles is very small (these experiments can be said the one that only has the reverse influence), the working principle of these instruments is the electromagnetic field of the particle to be influence on the medium in the instrument, the target particle just skimmed over at the regression center of the transverse section of the track. The inverse effect of the medium on the particle being tested is very small (negligible). Therefore, according to the above results (obtained by electromagnetic theory) we can know that the 3D regression curve of the cloud track of a charged particle in the chamber is moving trajectories of the measured particle in the past. This explanation is much more accurate and reasonable than the explanation of "micro particle dispersion interpretation in the whole track space". It can be said that the experiment of the cloud chamber and spark chamber to capture the motion path of high-speed particle has confirmed that the uncertainty principle is not universal (the paradox of uncertainty principle, see sections 6 and 7). The image process abut quantum decoherence is setting a man-made obstacles for quantum measurement. As long as the experiment using a cloud chamber to capture charged particles to meet the conditions of the quantum inverse measurement are recognized, the experimental results that have captured the tracks of motion particles have denied that the microscopic particles spontaneously and inevitably can overlap with their own shadow. Superposition between micro particles and their own shadow to be a common phenomenon has been denied by combination of the three factors (QIMT, the logic conclusion that superposition is mathematical possibilities but not the inevitability of physics and the experimental results to capture the track of charged particles). 
If the quantum inverse measurement is realized, we can find the real situation of microscopic particles under the Free State. The existing quantum mechanics theory holds that the quantum superposition state of free particle is never observed, can only be inferred by the results of the destruction of it. To insist on this point of view, we must deny the existence of quantum inverse measurement. In this section, the author points out the trapping of charged particles in a cloud chamber and spark chamber with quantum inverse measurement conditions. Some people have succeeded in observing the state of a single particle. The results of this kind of experiment together with the experimental results of quantum inverse measurement deny the existing quantum mechanics interpretation. In the existing theories of quantum mechanics, both "the state superposition" and "the collapse of the superposition state" are hypothetical and unverifiable unknown process (it is also a changing process at infinite speed that does not require time). "We'll never see the free state of microscopic particles", "the change of quantum state is instantaneous and super speed of light - can never know its specific circumstances and mechanisms". Is this explanation makeshift (improvising) or not? You know, something that will never be observed May not exist at least there are more than $50 \%$ possibilities. The superluminal process is a non real process. How reliable is it that the non real process whose possibility of occurrence has at most $50 \%$ ?

\subsection{The Measurement of Wave Function Unchanged and the Measurement That the Wave-Packet Collapse Cannot Be Caused}

For any wave function of the quantum system at a given moment, we can use the quantum Zeno effect to keep it constant, while simultaneous projection measurements of any observable amount will produce a definite measurement result, is the expected value of the measured observable quantity in the measured state. This measurement is called protective measure (Yakir Aharonov and Vaidman, 1993; Aharonov, Anandan and Vaidman, 1993).

The results of the secondary diffraction experiments of electrons show that continuous measurements do not result in the wave-packet collapse and quantum decoherence of micro-particles. The influence of the slit with more dense electrons on the incident electrons is not greater than the influence of the cloud chamber of the thinner vapor molecules on the incident electrons. According to this logic to judge, high-speed electronics in the cloud room is unlikely to be in the state of wave-packet collapse. Even if the high-speed electronics in the cloud room occurred in the wave packet collapsed and returned to the classic state, contradiction still exists. The $3 \mathrm{D}$ regression curve of the center coordinates of the droplets in the cloud trace of the electrons being measured in the cloud chamber is the precise trajectory of the measured particles (the position is accurate to reach the atomic size). In the microscopic world, if the position of the particle cannot be measured accurately to the atomic scale, there is an unreasonable phenomenon: in terms of position measurement granularity, classical mechanics and quantum mechanics upside down. Reason tells us that we should 
abandon the hypothesis about wave-packet collapse. The 3D regression curve of the center coordinates of the droplets in the cloud trace of the electrons being measured in the cloud chamber is the precise trajectory of the measured particles (the position is accurate to reach the atomic size). It is not difficult to see that in the electron secondary diffraction experiment, the state of the electrons changed before the electrons reached the phosphor screen, but the wave packet collapsed did not occur.

The state changes, but the wave packet collapse (quantum decoherence) does not occur, the quantum decoherence occurs, and the state does not change, and can partly meet the requirements of quantum inverse measurements.

\subsection{The Experiment to Measure Neutrinos}

Neutrinos are the smallest microscopic particles, and their quantum properties are more obvious normally. However, the general measurement is difficult to interfere with the movement of neutrinos. Almost all of the experiments on neutrino measurements are in accordance with the conditions of quantum inverse measurements.

\section{The Influence of QIMT on the States Superposition Principle}

The two reasons why the state superposition principle is used to describe object particles are: The nonlocal interpretation of the experiments results about quantum entanglements and electron diffraction experiments; object particles are made up of wave packets. These two reasons can be denied (the first is in Section 5.1, the second is in Section 5.2).

Quantum entanglement is one of the most famous predictions of quantum theory, theoretically derived from the principle of superposition. However, we have no reason to say that the superposition must occur. The superposition of entangled states must be nonlinear superposition, while the principle of superposition is linear superposition. This is also a contradiction. Since the process of quantum entanglement is unknown, Thus, what is currently we call "observed in the quantum entanglement process" is the things defined by the reporter's own, rather than the real phenomenon of quantum entanglement was observed. In Aspect and other experiments to verify the Bell inequality, the twin particle is entirely possible is always the pigeon pair (there is no experimental evidence to deny this argument). Before measuring the polarization state or spin state of the twin particles, we did not use the experimental method to deny that the twin particles are the pigeon pair (it is not validated by experiment that nay twin particles are not pigeon-pair). Under these circumstances, the process of proving the existence of quantum entanglement by experiment lacks a complete empirical chain. The empirical chain is also a logical chain. Therefore, it is also a logical loophole to test the Bell's inequality.

For the motion of particles, when you don't measure, you don't know where the moving track is, but it is not there is no definite track of motion. These concepts 
not only have a profound influence on the superposition principle, but also have a profound influence on the uncertainty principle. QIMT denies the mysterious effect of the unknown, and pursue establishment of the strict and complete logical chain and empirical chain. This makes it easier to discover the superposition principle and its application.

The superposition of the two possible states of the system is still a possible state of the system. This is a popular expression of the superposition principle. It explicitly states that state superposition is simply a mathematical permissible behavior, not an unconditional inevitable behavior. The superposition state is only "may be a kind of physical reality", not "must be the physical reality". It is a great mistake to regard possibility as necessity. Adherence to this error makes the interpretation of quantum mechanics cannot be a scientific explanation.

The quantum state of the complex system cannot be decomposed into the tensor product of the respective quantum states of the member system. Corresponding superposition is a nonlinear superposition, and the superposition principle is superposition of linear superposition. Both that a single particle superimposed with their own shadow and lost the original classic characteristics ant that twin particles are superimposed and lost their independence are also the process to be described mathematically rather than the necessary process in physics.

The most important part of this section is the proof that the evidence chain for quantum entanglement experiments is incomplete. The evidence chain (logical chain) is incomplete in the demonstration process of the conclusion of spooky action at a distance. It can be expressed in mathematical ways. But it is not a mathematical logical result.

\subsection{Empirical Train Incompleteness in the Experimental Verification of Bell Inequality}

This is also the insurmountable maximum logic vulnerability for the validation experiments of Bell's inequality. The reason is that the change in the quantum state of the twin particles is speculated by theoretical method rather than rigorously verified by experimental methods.

Logical chain is the logical "human reasoning path" or the main line of development of things, and is the string of a causal relationship (the interlocking strings of taking causal relationship as the main link). However, there must be an "empirical chain" when explaining experimental phenomena. "Empirical chain" means a rational chain formed by a series of sensory experience to be mingled with logical chain. "Empirical chain" is also an important part of the logical chain and evidence chain. Some experimental results are explained by quantum entanglement, in which the reasoning does not form a logical chain, especially the evidence chain has incomplete.

Only to observe a point cannot determine the speed. Only measuring the state of a particle on a point, we cannot be said to use experimental methods to prove the quantum state changes. When verifying Bell's inequality, we only measure 
the quantum state of the same particle at a point on. From an empirical point of view, we cannot say that the Bell's inequality is verified, the existence of interrelated phenomena is proved by the experimental method. The detailed discussion is as follows. The reason is that the change of quantum state has not been experimentally verified (the situation that twin particles are the "pigeon pair" from beginning to end must be ruled out, but we did not use the experimental method to exclude). This is the incomplete of empirical chain. If empirical chain is incomplete, the logical chain is also incomplete.

If we want to determine the change of state by experimental method, we must use the experimental method to measure the difference between before and after the two states. However, the existing quantum entanglement experiment only measures the states of twin particles after the change, and the states before the change are not measured by the experimental method. The particle state before measurement is assumed (or just inferred from the theory). The change of the quantum state has not been experimentally verified, and the super correlation derived from the quantum state change has not been experimentally verified. Visible, in the process of interpretation of the measurement results of quantum entanglement, the so-called "the change of quantum state" is very likely to be that the quantum state does not change at all. Concretely speaking, the explanation of quantum entanglement experiment made two mistakes: first, it is wrong to think that the homologous conjugated particles must be in an entanglement state (it is also a superposition state or a mixed state) before the observation; second, it is wrong to think that even interference-free measurement, the quantum state can be changed. It is recognized that the entangled state existed before the experiment. After the experiment, it was admitted that the existence of quantum entangled state have been proved. This is obviously a logical loop. The first wrong understanding is just a hypothesis, has never been directly confirmed by the experiment, it cannot be confirmed by the experiment. Entanglement interpretation of quantum entanglement experiment depends on the first error. The first mistake is to assume (or cognizance) that the particles are in an entangled state before being measured (the entanglement state of homologous conjugated particles is the superposition state or mixed state). The purpose of quantum entanglement experiment is to prove the existence of quantum entanglement, but the explanation of the experiment must use the assumption that the quantum entanglement exists before the measurement. This process obviously belongs to a kind of circular argument. Professor Ronald Hanson's experiments [19] did not completely rule out the most critical logical vulnerabilities in the John Stewart Bell experiment.

As described above, in the verification experiment of Bell's inequality (or Alain Aspect experiment), the change of quantum state has not been proved by experiments, and super-correlation between twins particles can only be derived from the change of state. Therefore, the demonstration (interpretation) process of the experimental results does not form a complete empirical chain and a complete logical chain. 
Only the measurement of information reverse transmission is equivalent to no interference measurement. The measurement of free particles without interference can be realized by quantum inverse measurement. The true colors (true state, to be also the state of reality) of micro particles can be observed by quantum inverse measurement. Since the measured object is not subject to the interference of the observer, the presence or absence of the observer is independent of the motion state of the measured object (the coupling between the measured object and the observer cannot occur). Since there is no coupling between the measured object and the observer, there is no need to segmentate them. In other words, in the process of quantum inverse measurement, "this important 'Archimedes segmentation point' of segmentation of the observer and the observed object can be obtained".

Taking an electron of free movement as an example, if it being at $A$ but not at $B$ has observed by an inverse measurement, then the mixture state that it is both at $A$ and at $B$ (at the same time, the electrons are both at $A$ and at $B$ ) cannot be observed. Maybe someone will say, in the diffraction experiment by double slit of electron, that an electron can be simultaneously located at $A$ and $B$ have observed, isn't it? However, the previous electron diffraction experiment is not a quantum inverse measurement experiment (because the instrument has a serious interference with the target particles as measured). Moreover, the results of electron diffraction experiment can be explained with the viewpoint that the effect of incident electrons leads to the generation of photons, and the diffraction fringes are caused by the accompanying photons (called accompanying photon effect for short). Electron diffraction experiments have not ruled out this possibility at that time. Since there is no rule out this possibility, tough to adopt the interpretation that electron have wave character is not strictly. The diffraction of photon pass through double slit is not explained by that a photon is both at $A$ and at $B$, but using the Huygens principle to explain. Visible, even if the electrons are completely waves, the results of the diffraction experiment by double slit of electron do not necessarily prove that at the same time, the same electron both can be at $A$ and can be at $B$. it could not prove that the electron is in a superposition state: The entity of the electron at $A$ is superimposed with its shadow at $B$. The superposition state of an electron with its own shadow is a state of non reality. It cannot be observed by quantum inverse measurements. In the concept of orthodox quantum mechanics, the superposition state of an electron with its own shadow can also not be observed (as long as the measurement, this state is destroyed).

Put a cat in a closed box, and then connect the box to a device. The device contains an atomic nucleus and a toxic gas facility. The atomic nucleus has a fifty percent chance of decay, and a particle will be emitted when the nucleus decays. The particles will trigger the poison gas facility, so as to release the poison gas to kill the cat. This is the famous Schrödinger dead-live cat state of thought experiment [20].

For this thought experiment, the past three errors are: First, the way of obser- 
vation is confined to open the box observation; Second, it is considered that the resistance of the quantum superposition state is zero, any observation and measurement can destroy the quantum superposition state; Third, it is erroneously assumed that macroscopic objects can also be superimposed in quantum states. Quantum physicists set up an unproven observation barrier. Then take the artificial obstacle as the premise, and derive the conclusion that purely objective quantum states cannot be observed, and the observed ones are newly produced in observation. This is a thinking trap, and is also a mud pit of agnosticism. We must bypass, and can also bypass. In fact, in the thought experiment of Schrödinger cat state, observation of the cat can also take the way of inverse measurement (only the signal is transmitted from the cat to the viewer and no signal is transmitted from the observer to the cat). One of the concrete methods is to put an infrared receiver's probe into the box where the cat is in. The outside observer is only looking at the display screen of the infrared receiver. Through this screen you can see whether the cat is standing or falling down. The whole process from the cat into the box to open the box can be photographed by the infrared camera. Regardless of whether the observer to see the display screen, cannot interfere with the status of the cat, and can promptly know the cat inside the box is dead or living (Standing or falling). The observer also known that the nucleus has decayed or has not. Another method is attached the auscultation head of a stethoscope to the cat's chest, the hose extends to the outside of the box and is connected with the earplug, the experimenter listens to the cat heartbeat in the box. Before people just think of the way to open the box, really too rigid. For macroscopic objects, quantum state superposition is not possible. Therefore, the mixed state of the dead-living cat cannot be an objective existence for real observation, and it's also not to be seen. As long as the mixed state of the dead-living cat does not exist, the superposition between decay state and nondecay state of the nucleus does not exist. The Subjective Intervention Concept that the ideological consciousness of an observer wanting to observe the cat state has disturbed the cat state doesn't have enough bases.

The complete Schrodinger cat state thought experiment will convert the measurement of quantum states of microscopic particle into the measurement of macroscopic objects. While the measurement of the macro object is easier to achieve the operation that the instrument has no interference to the measured object. In fact, it is theoretically possible to deny the existence of the superposition state by transforming the observation of the microscopic state into the observation of the macroscopic state of the object. The reason is that quantum states do not superimpose, for the macroscopic objects, and do not appear to overlap with their own shadow.

In this experiment, the measurement of microscopic particles is transformed into the measurement of macroscopic objects. For macroscopic objects, it is easier to realize the measurement interference-free (or the interference can be ignored completely).

As mentioned above, the superposition state of the microscopic particles with 
their own shadow is the non-real mathematical virtual state which does not exist and is not observed. By using the quantum inverse measurement technique, we can make Schrödinger using his cat state experiment to achieve his desired objectives. At least we can use the quantum inverse measurement experiment to check whether there is this kind of non-real mathematical virtual state.

\subsection{The Contradiction of the Stability of Superposition State in the Interpretation of Quantum Entanglement}

This is also the contradiction between the explanation of continuous quantumentanglement experiment and the explanation of instantaneous quantum-entanglement experiment.

In December 2005, in the journal of Nature, D. Leibfried, E. Knill, et al. reported that the entanglement was sustained by $50 \mathrm{~ns}$ [16]. For convenience, this type of experiment is called a continuous quantum entanglement experiment (continuous quantum entanglement is observed by it). The experiment, such as Aspect et al., Which tests the Bell inequality, is called the instantaneous quantum entanglement experiment (the instantaneous quantum entanglement effects are observed by them). In the quantum entanglement state of $50 \mathrm{~ns}$ duration, the measurement must also only be uninterrupted continuous measurement. In other words, the measurement of the "The entanglement lasted for some time" requires that the measurement (observation) is also continuous and that the measurement (observation) operation does not destroy the quantum state (without causing the wave packet to collapse). This measurement is in accordance with the quantum inverse measurement condition: purely objective measurement result can be obtained by the measurement that the interference can be ignored. It can be seen that this experiment to capture the Schrödinger cat' state actually supports QIMT. The interpretation of the superluminal correlation between two twin particles in the entangled state requires the premise of "as long as the measurement, it will destroy the superposition state (wave packet collapse)". It is obvious that the explanation of the sustained entanglement of the Schrödinger cat state experiment and the "superluminal correlation of entangled particles" in the Alain Aspect quantum entanglement experiment are contradictory: if it is "As long as measuring wave packet will collapse", continuous entanglement cannot be observed; if it is "measurements may not result in collapse of wave packets", we cannot use the explanation of "there is superluminal correlation between twins particles". People do not know exactly what state of quantum entanglement is. After the alternating change of the spin direction (or the alternating change of the polarization direction) is measured, it is believed that this is quantum entangled state. However, admitting this is to admit that the twin particles were pigeon pair. If the twin particles are recognized as pigeon pair, the existing quantum entanglement experiments have become problematic. The "boy" and "girl" in the pigeon pair intertwined (especially, the "boy" and "girl" in a single particle intertwined) has no strong experimental basis.

Measurement action (or instrument) of the continuous quantum entangle- 
ment experiment does not destroy quantum entangled states. Admitting the results and interpretations of their experiment, it is recognized that the measurements do not change the superposition state of the microscopic particles, i.e., the measurements do not result in the collapse of the wave packet. If it is admitted that the entanglement can be sustained during the measurement, it must be recognized that the measuring instrument and the observed object can be segmented in this duration (supporting the existence of quantum inverse measurements). However, in order to obtain the instantaneous quantum entanglement experimental results, it was previously believed that as long as the measurement, the quantum superposition state, especially the quantum entangled state, will be changed (destroyed). If we do not recognize the change, we cannot recognize the quantum entanglement to have been found. This is a fatal contradiction in the interpretation of quantum entanglement experiments: as long as the measurement will change the quantum state, must not be able to continuously measure the quantum entangled state; if the measurement cannot change the quantum state, many of the so-called quantum entanglement experimental results (e.g. Aspect's experimental result) cannot show that the mysterious correlation between the twin particles. It has been argued that both quantum entanglement and quantity teleportation are philosophical rather than physical explanations. That is, quantum entanglement is a psychological product rather than a real physical process.

The thinking of the interpretation of instantaneous quantum entanglement experiment is: before measurement: the spin state of the twin particles is uncertain $\rightarrow$ Measurement leads to collapse of the wave packet $\rightarrow$ The spins of the twin particles were simultaneously measured to be opposite (there is a mysterious association between them) $\rightarrow$ Conclusion (there is spooky action at a distance). The thinking of the interpretation of continuous quantum entanglement experiments is: Continuous measurement $\rightarrow$ the continuous entanglement of twins is measured $\rightarrow$ Conclusion (measurement does not destroy the quantum entangled state, and measurement does not lead to wave packet collapse). Obviously, these two kinds of experiments are obviously contradictory: for the former, measurement must lead to the quantum state change; for the latter, continuous measurements do not lead to quantum state changes. If these two kinds of experiments are connected in series (continuous is in front, instantaneous is in the behind), scientists will not be able to explain the experimental results. QIMT can eliminate this contradiction. If you do not recognize the contradiction between the two experiments, it is not logical, and the existing interpretation of these two experiments is not science.

\subsection{The State Superposition between Particles Is Only “Allowed to Happen" in Mathematics Rather Than the "Inevitable" in Physics; Even If the Superposition, There Are Differences in Degree and Efficiency}

The overlay between an individual particle and its shadow is a low probability 
event. Twin particles are the pigeon pair; at the beginning. The physical state that most match with mathematical state-superposition is the superposition of empty states. In addition, we must consider the superposition efficiency. The superposition efficiency includes the intensity of the interaction between the various parts involved in the superposition or their contribution to the spatial point. The next section shows that the object particles are localized. The superposition efficiency between the localized particles is related to the distance between them (or the distance of the center of gravity of each part from the point of consideration).

Superposition principle is a basic principle in quantum mechanics. It illustrates the nature of the wave function. If $\psi_{1}$ is an intrinsic state of the system, the corresponding eigenvalue is $A_{1}, \psi_{2}$ is one of the intrinsic states of the system, and the corresponding eigenvalue is $A_{2}$, according to the linear relationship of Schrödinger equation, $\psi=\mathrm{C}_{1} \psi_{1}+\mathrm{C}_{2} \psi_{2}$ is also a possible existence state of the system $\left(\psi=\mathrm{C}_{1} \psi_{1}+\mathrm{C}_{2} \psi_{2}\right.$ is the one of the form of expression of Equation (5)). If you measure the observable quantity $A$ in this state, the $A$ values to have measured are both likely to be $A_{1}$ or $A_{2}$, and the corresponding probability ratio is $\left|C_{1}\right| /\left|C_{2}\right|$. The average value of $A$ in three dimensional full space is $\langle A\rangle=$ $\int \psi^{\star} A^{\prime} \psi \mathrm{d} x$ or the Dirac symbol $\left\langle\psi\left|A^{\prime}\right| \psi\right\rangle$. The ratio of the probability to $\left|C_{1}\right| /\left|C_{2}\right|$ is the theoretical source (theoretical basis) of the quantum mechanical probability interpretation.

In the above statement, "if $\psi_{1}$ is an intrinsic state of the system, $\psi_{2}$ is also an intrinsic state of the system" can be used to describe empty states. However, if we use it to describe real states and recognize that a system can simultaneously be in two states, we admit that a system can simultaneously be in these two states. It is equivalent to admitting that a person has two faces at the same time, and these two faces are his real face, because the eigenstate is a state of full representation, not a partial state. If the objective real face of a particle (or a person) is only one, then, there must be one in the $\psi_{1}$ states and $\psi_{2}$ states of microscopic particles that is fictitious (or spare/alternate). The idea that a particle simultaneously has two different real faces was based on supposition (hypothesis). This hypothesis is also a hypothesis that microscopic particles have non localreality. Interpretation of the experimental results of double slit diffraction of electron does not rule out the accompanying light effect of most likely to occur, and it cannot be served as a solid evidence of an object particle simultaneously having two different real faces. In addition, in the above statement, the person to propose state superposition principle firstly recognizes that the states superposition at first was just a possibility. The next word "the $A$ values to have measured are both likely to be $A_{1}$ or $A_{2}$, and the corresponding probability ratio is $\left|C_{1}\right| /\left|C_{2}\right|$ " recognized the state superposition to be inevitable (if the superposition does not occur, the measurement results are not statistical). For the superposition of states, there is a lack of necessary logical transition from "possibility" to "inevitability". State superposition is also an inevitable (hypothetical). 
It can clearly be seen that, if the state superposition principle is used to describe the real state of individual particle, there are two virtual things: microscopic particles are made up of wave packets, or "a particle has two different faces"; the superposition between the first face and the second face of a microscopic particle is necessary. From a scientific point of view, the "principle" containing two fictitious is not strict. The experiments using cloud chamber to display the motion track of charged particles have proved that the charged particles are not two different faces. The superposition of the two different faces of a particle is sheer fiction. QIMT is not optimistic (criticism) about the state superposition principle based on the two imaginary. The above statement shows that, whether according to the theory or according to the experiment, we don't have enough reason to deny "the existence and realization of quantum inverse measurement". Von Neumann theory is derived from the mathematical method of Hilbert space operations. He also did not prove that the possibility of mathematics must be the inevitability of physics. Fictitious just is the hypothesis and speculation. Therefore, the superposition of quantum states can only be assumed. In the micro world, whether such a hypothesis is generally true is a problem. There is no good reason to raise it to the height of the principle. Denied the superposition of quantum states also denied the interpretation of quantum mechanical probability.

The outer layer of the carbon atom has $2 s$ and $2 p$ electrons. Before they are hybridized, $2 p$ electron is not another eigenstates of $2 s$ electron, and $2 s$ electron is not another eigenstates of $2 p$ electron. The eigenstates of $2 s$ electron is the electron movement state that the $2 s$ electron is in the $2 s$ sub layer (only an eigenstate). Whether the $2 s$ orbit and the $2 p$ orbits of the carbon atom are hybridized or not, and what form of hybridization must be determined according to the conditions. Electronic orbital in gaseous carbon atoms are not hybridized; under the condition of low temperature and pressure, the carbon atoms form graphite in the form of $s p^{2}$ hybrid; in the high temperature and high pressure to form $s p^{3}$ hybrid diamond.

If the physical particles are not discrete but localized, they farther away from each other, they superimposed degree is lower, and the superposition efficiency is lower. The superposition can be ignored when the degree and efficiency of stacking is reduced to a certain extent.

The mathematical possibility is not the inevitability in physics. Not all of the coupling has a very high degree and efficiency. If there is no coupling between the measured object and the observer or the degree and efficiency of the coupling is very low, there is no need to divide them. One of the serious mistakes that quantum mechanics has made is to regard mathematical possibilities as a physical necessity. This behavior is very not dialectical.

A branch of mathematics called Hilbert space. The theory of quantum mechanics can be constructed by the mathematical construction of Hilbert's space. Both the state superposition expressed by Equation (9) and the coupling expressed by Equation (11) are derived from the mathematical method of Hilbert 
space. The wave packet collapse model of von Neumann [21] was regarded as the standard model of quantum measurement. Its main idea is that if we want to measure a certain mechanical quantity of quantum system, we must consider the function of the measuring instrument, and use the language of quantum mechanics to describe it. This model is that the mathematical possibilities of the coupling described by the Hilbert space are regarded as the inevitability of quantum mechanics ("we must also consider the function of the measuring instrument" admit "the coupling is inevitable"). However, as this article has been explained, in the process of quantum inverse measurement, the observer has no sufficient effect on the observed object, and the coupling between them will not occur. Under the background of QIMT, new measurement view is "under certain conditions, in order to take into account the role of the instrument".

The concept of quantum entanglement thinks that the original superposition (or entanglement) of the microscopic particles expressed by Equation (9) is unconditional, there is no interference free measurement, the measurement with interference is bound to destroy (change) the original superposition state. If a non superposition state of the microscopic particles has been observed by using the quantum inverse measurement method, it is indicated that the non superposition state is the intrinsic state of the measured particle. The realization of the quantum inverse measurement is to realize the measurement of no interference to the observed object, and such a measure of action does not change the original superposition state of the measured particle. If the quantum inverse measurement is realized, not only it indicate that the coupling between the instrument and the observed particle have not formed, but also indicate that there is no sufficient reason for the superposition between the particle and its shadow (there is no reason for the possibility of mathematics must be the inevitability of physics). The existing significance of the experiment on quantum entanglement and the verification of Bell inequality are questionable [22] [23] [24].

If we believe in the existing explanation for the experimental results of quantum entanglement, we must first admit the existence of quantum entanglement expressed by Equation (9), then it can be said that the quantum entanglement has been measured by experiment. If we do not admit the existence of quantum entanglement expressed by Equation (9) (do not take the possibility in mathematics as the inevitability in physics), the experimental results cannot be interpreted as "the quantum entanglement to be observed by the measurement". It can be seen that there exist logical cycles in the interpretation of the results of the quantum entanglement experiment. If there is no original and spontaneous superposition of quantum state, there is no concept of quantum decoherence and wave packet collapse.

In short, the superposition of quantum states is only a mathematical possibility, not the reality inevitable. The states superposition is conditional, not unconditional. The wave packet collapse caused by observation is conditional, not unconditional. It is a kind of sophistry needs that take the possibility in mathematics as the inevitability in physics. 


\section{The Fresh Blood Is Inputted for Quantum Mechanics by Light-Knot Particle Structure Model}

There is an association between the three: the principle of states superposition, the wave-particle duality of matter particle and the uncertainty principle. It can be said that they are bound together for good or ill. Letting electrons pass through a slit is not a good way to measure the position and momentum of an electron simultaneously. QIMT allows the presence of interference free measurements and allows for the presence of non random interference measurements. Both the interference-free measurement and the non-random interference measurement can obtain the pure objective state of the measured object, and the uncertainties of the microscopic particles mentioned earlier are also absent. In this section, we first comment on the quantum mechanics measurement method, then talk about the electronic structure model of light junction, and finally talk about the influence of QIMT on the uncertainty principle.

The first two sources of uncertainty theory are Heisenberg's presentation and Earl Kennard's presentation. Described in modern languages, these two statements are: $<1>$ measurement inevitably and irreversibly destroyed the state of quantum; $<2>$ the inevitability of superposition of quantum states (or microscopic particles with wave-particle duality) determines that the uncertainty of microscopic particles is primitive and spontaneous. Now, it is believed that, in the determinants of the uncertainty principle, there is still: $<3>$ explanation of electron diffraction experiment; $<4>$ according to the principle of quantum mechanics, the mathematical expression of uncertainty principle can be derived. The emergence of QIMT can make people more clearly understand the problems of the four determinants.

We will introduce the following: the direct influence of QIMT on the uncertainty relation; the other problems of the principle of uncertainty; the best method to measure the position and momentum of particles is introduced.

\subsection{Both the Projection Measurement to Use Photons to Influence the Observed Particles and the Measurement to Use Slits Are Not the Best Measurement Method}

When Heisenberg put forward the uncertainty principle, the quantum measurement method enumerated by him was a projection measurement method (comparison with inverse measurement, it belongs to forward-inverse measurement). This is an unreasonable measurement method for measuring microscopic particles. Measurement of microscopic particles is best use the method that the electric field of measured particle is unilaterally received by the measuring instrument (i.e., an inverse measurement method). There are other protective measurement methods. It is difficult to obtain a universal conclusion by using the projection measurement method.

The direct meaning to reveal the paradox of uncertainty principle is to know that the past motion path of a microcosmic particle can be accurately measured when it is continually measured. This conclusion can be verified by the experi- 
mental method of continuous measurement [24]. Von Neumann's quantum measurement standard model cannot be used to completely eliminate the paradox of uncertainty principle.

The uncertainty of microscopic particles originates from superposition of states. For a particle far from the environment and other particles, it is only superimposed with its shadow. As long as the particle is not a ghost that has independent consciousness and/or spooky action can occur, it has no legitimate reason that free particles are not certainty.

The best way to measure the electron position and momentum is to observe the motion trajectory of electrons with a cloud chamber. This method was not used before. The reason is that, in the same time accurately measure the position and momentum of the electronic case, and the Copenhagen school does not recognize that it is also accurate measurement of the electronic momentum and position. The school argues that electrons may appear in the space occupied by the entire cloud track. So there is a logical problem: while recognizing the collapse of wave packets while recognizing the superposition of electron and space hole (two states superposition leads to electron in the dispersion state); the space occupied by an electron is not the measurement uncertainty of the electron position. Therefore, the thickness of the track is not the measurement uncertainty of the electron position. The electron track measured in the cloud chamber does not correspond to the strict definition of uncertainty relation. Orthodox quantum physicists believe that electrons passing through the chamber and the wave packet will collapse, the observed electron returns to the classical state. However, they still believe that the motion electron in the cloud chamber is dispersed in the space of the whole cloud track. Dispersion is produced by superposition of quantum states. Now that the wave packet collapses and the quantum superposition disappears, how can it still diffuse? Even it is diffuse, the physical meaning of the 3D regression curve of the droplet center coordinates in the cloud track should also be the trajectory of the center of gravity of the measured particle! We have no way to rule out "the 3D regression curve of droplet center coordinates in the cloud track" is the exact movement route of the electron.

\subsection{The Electron Structure Model for Light-Knot Can Reduce the Number of Quantum Mechanical Postulates and Can Reveal That Sources of Probability Are Not Reliable}

A circularly polarized photon propagates along a closed path to form an electron or a proton. This kind of particle structure model is called the light knot model of object particles. This model shows that the essence of the object particles in the wave-particle duality is that the whole of the object particle has the characteristics of classical particle, and the reason that the object particles are volatile (to can be described by the wave function) is that the particles are surrounded by waves. This wave-particle duality does not determine that the particles are non local-real (discrete). There is a good unity between wave and particle that has not been used before. Wave-particle duality is the combination of two characte- 
ristics and performance in one, which does not mean that the whole of a particle is discrete.

The influence of state superposition on particle uncertainty and the influence of wave-particle duality on particle uncertainty are in the form of different but the same result. The problem of superposition of quantum states has been discussed above, so this section focuses on the problem of wave-particle duality. The erroneous ideas of quantum mechanics are due to the lack of understanding of the composition and structure of microscopic particles. To correct the wrong idea of quantum mechanics, one is to proceed from the theory. The two is to start with the structure and composition of micro particles. In this section, we briefly introduce a kind of electronic structure model of light knot. The wave function of electrons is determined by this structure.

The uncertainty principle must depend on: the original spontaneous superposition of state of microscopic particle is widespread and spontaneous; the measurement destroys the quantum superposition state. The existing quantum mechanics scientists think that after the first measurement, the superposition state of the measured particle has been destroyed and has returned to the classical state, and cannot return to the original quantum superposition state again. That is to say, we can't measure continuously under without destroying the quantum superposition state. Measurement of particle system without interference has been realized by quantum inverse measurement. There is no limit to the above. We can measure a microscopic particle continuously without interference. For the measurement in accordance with inverse measurement conditions, the measured trajectory is not caused by the dispersion of the particles, usually caused by particles emit electromagnetic field signals.

Logically, the particles that can be stationary are localized particles, the particles that cannot be stationary are discrete waves, and the discrete wave propagates along a small closed path to form a localized particle. This is the structure of the wave knot of fundamental particle. For electrons or proton, the "wave knot" is a "light knot". Closed chords are also of this structure. At the beginning of the establishment of quantum mechanics, Lord Kelvin mentioned the elementary particle structure model. But he mistakenly believes that this structural model cannot solve the problem of atomic stability, so that the model has not been recognized. Kelvin is too famous, he has no confidence in his theory, others will not support. Not the model is incorrect. In the 21 st century, whether it is superstring theory or loop quantum theory, as well as geometric algebra material structure theory, in essence, they are based on the quantum motion - closed curve of the knot.

In Ref. [1], I point out that an electron is formed by a simplest circularly polarized photon propagating along the closed path (belonging to a closed string structure model: is the one kind of Kelvin wave model of the knot). The wave function of circularly polarized light is

$$
\psi(x, t)=a \mathrm{e}^{-i 2 \pi(v t-x / \lambda)}
$$

The wave function of electron is also (12). It also shows that an electron wave 
is a real monochromatic wave rather than a probability wave or a wave packet. The square of the module of the amplitude is field strength rather than probability density. The whole of this light-knot is lacalized. The center of gravity of the object particles can still be described by position and velocity, and the future of moving particles can be predicted. This structural model laid the foundation for the establishment of interpretation system of quantum mechanics of local-realism and determinism. The experimental results of diffraction by double-slit of electron can be explained by the effect of accompanying light. After reading this passage, you should be more convinced that the $3 \mathrm{D}$ regression curve of the cloud belt in the chamber is the exact path of the particle.

The reader may have noticed that I replaced "the wave function in the general textbook to be similar to Equation (12)" into "to be Equation (12)". This is not just a word problem, but there are essential differences. First, I pointed out the source of the wave function. Second, a variety of operators can be derived from Equation (12). The method is as follows, to do partial differential operation of $q$ and $t$ for Equation (12). Both the first order partial differential and the second one are required. According to the classical formula of mechanical quantity, $p=$ $h / \lambda($ or $m v=h / \lambda)$, the bound motion equation of a charge, the fine structure constant expressions, the electron velocity in ground state hydrogen atom $v=\alpha c$ obtained by Bohr's atomic model, we can get the corresponding mechanical quantity operator. The eigenvalues of the corresponding mechanical quantities can be obtained by applying the resulting operator to Equation (12). According to $E_{p}=p^{2} / 2 m, E=E_{p}+V$ and the above method, energy operator can be obtained. The energy operator acting on the wave function is the Schrödinger equation [1]. It can be seen that the third postulate of quantum mechanics is not the most fundamental, but can be deduced. Third, the eigenvalues of the mechanical quantities thus obtained are unique, not probabilistic. Fourth, it is finally shown that the state after the measurement can still be described by the Schrödinger equation. Is the four, completely destroyed the existing quantum mechanics measurement concept, and smashed the cornerstone of the quantum mechanics for non local-realism.

$$
\hat{A} \psi(x, t)=A \psi(x, t)
$$

The operator $\hat{A}$ of mechanical quantities is arbitrary. If the operator acts on the wave function is equivalent to the measurement, then Equation (13) means that the state of the system is not changed by measurement (the wave function $\Psi(x, t)$ on both sides of the equal sign $(=)$ is exactly the same). The premise that the electron consists of the simplest circularly polarized photon determines that the wave function described by Equation (12) is very stable. If it does not meet the violent conditions of the annihilation or decay, its form will not change. If you do not achieve the above two conditions, the other actions are equivalent to the actions of the field to the wave. These effects follow this law that, in the potential field, the energy of the wave changes but the form of the wave does not change. In other words, measurements under non-violent interactions result in 
only energy changes without causing structural changes in particles (the structure has not changed, of course, the nature has not changed). Change in energy, the speed of motion and the direction of motion (and perhaps the direction of spin) can be caused by measuring. The main content of the electronic structure model for light knot is that the wave make a circle is the particle, and the particle nature is the wave. When a wave changes into a particle, it does not collapse. The movement of particles is the overall movement of light knot, not the movements that appear and disappear mysteriously. When it is not measured, it conforms to the laws of motion of Newton and the laws of electromagnetism (the states of particles are definite). In other words, as long as the particle is objective, it is definite (with definite form of existence and motion) before it is measured.

The closed-string structure of the localized particle is particle structure which is most coordinated between the discrete wave and the local particle. This particle structure denies the existence of the point-particles, affirmed the object particles are also waves, and explained the wave-particle duality of object particles. If you follow the past concept of particles, this structure is the unity of particles and waves. Although the particles are formed by waves, the whole of a particle is localized and has a center of gravity. Since the particles are formed by waves, it is not surprising that the particles have wave-particle duality.

A 3D regression curve of droplet center coordinate can be obtained by using cloud chamber to capture a high-speed moving charged particle. According to the working principle of the chamber we know that, Even if "a measured particle returning to the classical state due to the collapse of the wave packet" is true, this curve can only be an accurate moving path of the measured particle. The secondary diffraction experiments of electrons show that continuous measurement can be made under conditions that ensure that quantum coherence does not disappear. If we believe that the light-knot electronic model, then the $3 \mathrm{D}$ regression curve caused by the measured electron is the curve drawn by the movement of the gravity center of the light knot. The thinking to infer this conclusion is very clear. Moreover, it is pointed out that the object particles are not wave packets, and the state obtained by means of measurement is not the state of the collapse of the wave packet but the original state of the particle. In this case, the $3 \mathrm{D}$ regression curve mentioned above can only be the exact path of the particle. Even if the particle is composed of a wave packet, the 3D regression curve is also the line drawn by the center of gravity of the wave packet.

\subsection{The Results of Electron Diffraction Experiments Are Not Conclusive Evidence of the Principle of Uncertainty}

The first experimental evidence of the uncertainty relation is the electron diffraction experiment. However, as long as we carefully analyze, the diffraction experiments of electrons and other object particle are not the experimental basis of uncertainty relation. The choice of the Copenhagen interpretation needs to rule out a possibility, but it has not been ruled out. This possibility is that the diffraction is caused by the excited photons. That is, the diffraction of the object 
particles is a side effect, and the positive effect is the performance after the socalled wave packet collapse.

When the results of electron diffraction experiments are taken as the basis of the uncertainty relation (in the process of according to the experimental results of electron diffraction derived uncertainty relations), measuring the position of the electron and measuring the direction of movement of the electron are not simultaneous: the moment at which the position of the electron is measured is the moment when electron pass through the slit; the moment to measure the direction of the electron is the two time that the electron hit the screen and the electron pass through the slit. From this we deduce the direction of motion of the electron, and we must admit that the electron is in a straight line between the slit and the screen. For a single measurement of an electron, admitting that the electron is in a straight line along the direction of the determination equals admitting that its position and direction of motion are determined. In this way, between the slit and the phosphor screen, the position and the direction of movement of the measured electron have a certain value at the same time. This result does not support the uncertainty relation. Recognizing that the measured electrons take a straight line during this time, there is another problem: The collapse of the wave packet must occur before the electron passes through the slit and reaches the screen, and the diffraction pattern should not appear. However, the diffraction pattern actually appears. This is a contradiction, or logic loophole.

Letting a particle passing through a slit is not a good way to measure the position and momentum of a particle. The measurement uncertainty $(\Delta x$ and $\Delta p)$ obtained by this method is not a universal limit measurement uncertainty. Why we cannot accurately measure, the reason is because the method is not right. According to QIMT, it is considered that the best method of measurement is the measurement of interference $=$ free (or the interference can be ignored). In the electron diffraction experiment, the slit width (or pinhole diameter) $\Delta x$ is not the measurement uncertainty of the position (it is intuitive that the length of the rectangular slit is not the measurement uncertainty of the position of the incident particle). Just as the airplane passes through a bridge hole, the size of the bridge hole is not the measurement uncertainty of the aircraft position. $\Delta x$ is a man-made space constraints, its value reflects the strength of the interference. The measurement uncertainty of position is a statistical value. $\Delta p$ is also not the measurement uncertainty of a momentum. We let a large number of electrons through a small hole to get a concentric circular diffraction pattern, and then one by one to send electrons through the hole. We examine exactly the electron on the center of the original concentric circle. We examine the electron to have reached at the center of the concentric circle. The uncertainty of the direction of the electron is mainly determined by the measurement error of the deflection angle and the measurement error of the electronic velocity.

The momentum uncertainty calculated by the theory and method of uncertainty (JJF 1059.1-2012 or GUM: ISO/IEC Guide98-3-2008) is not the $\Delta p=$ 
$m u \sin \theta$. Only after the numbers of the same electrons were measured, the obtained standard deviation is $A$ type of uncertainty, and its value is also not equal to $m v \sin \theta$ (it is certain that it is less than $m v \sin \theta$ ). Moreover, the position of the electrons emitted by electron gun passing through the small hole each time is not the same. In this case, the $\Delta p=m v \sin \theta$ is caused by the interaction between the electron and the slit, rather than the uncertainty of the momentum of each electron passing through the center of the hole. According to the quantum inverse measurement concept that the particle beam will not be deflected when the measurement is interference-free (or interference is very small, or interference is very balanced), the electrons that hit the target center are also through the center of the hole, and the electron traveling in the direction parallel to the electron gun are also emitted from the center of the aperture of the electron gun. We can also determine whether this electron really passes through the center of the hole by observing whether the three points (ejection center, the center of the hole and the target point of this electron) are in a straight line or not. If it is, it indicates that the electron locates in the center of the orifice. The measurement uncertainty of the position of the electron passing through a small hole is determined by the measurement error of the spot diameter on the screen, the measurement error of the electron gun caliber and the measurement error of the deflection angle of the straight line, and it is also not the aperture (or slit width) $\Delta x$ of the small hole. At this point, the degree that the electron beam deviation from the center of the small hole is caused by the deviation of the emission direction of the electron, rather than the measurement uncertainty of the position of the electron. When $\Delta x$ is large, it is not the case that the measurement uncertainty of the particle position is more pronounced. Therefore, we conclude that the experimental results of electron diffraction do not show that $\Delta x \Delta p \approx \hbar$ is the relation of measurement uncertainty. It can be a mathematical relationship of $\mathrm{AB}=\mathrm{C}$.

\subsection{The Past State of a Particle Can Be Measured Accurately}

Heisenberg used an erroneous testimony about uncertainty relation (the cited examples are projection measurement that the interference cannot be eliminated). It does not have universal significance. Under the constraints of misinterpretation, the present situation is that even if the position and the momentum of a microscopic particle are accurately measured at the same time, they are not recognized. The relationship between nonlocalization and uncertainty relation is contradictory.

If we continuously measure the position of a particle in a flight applying the way of quantum inverse measurement, both QIMT and the uncertainty principle allow the position of the particle to be continuously measured accurately. So, as long as the position of the particle is accurately measured continuously, the motion track of its past can be painted by according to these position points (that is, its past track has been accurately measured), the instantaneous velocity and motion direction of its past are also accurately tested. Logically, if the position of a moving particle in space is continuously determined, its past momentum cannot 
be uncertain. This is the logical paradox of the uncertainty principle (the paradox of the uncertainty principle for short). It is revealed by QIMT. If a microscopic particle is delocalized, then, both its position and its momentum cannot be accurately measured rather than just one of them can't be measured accurately.

As we all know, the principle of uncertainty allows the one of the two conjugate physical quantities of a moving particle to be measured accurately enough. There is a brief mathematical proof as follows. At the time interval of $d t$, the position of a moving particle is measured twice. If both the positions of the particles are measured sufficiently and accurately at these two moments, and they are $\left(x_{1}, y_{1}, z_{1}\right)$ and $\left(x_{2}, y_{2}, z_{2}\right)$ respectively, the distance between them is small enough $|d r|$, then, in this interval, the past movement direction of the particle is accurate $d r / d r$, the accurate momentum of the classical mechanics of the particle in the past is $m d r / d t$. Visible, at the point $\left(x_{1}, y_{1}, z_{1}\right)$ (or between the two points $\left(x_{1}, y_{1}, z_{1}\right)$ and $\left.\left(x_{2}, y_{2}, z_{2}\right)\right)$, both the position and momentum of the particle in the past can be simultaneously measured accurately enough. The connection between the two points $\left(x_{1}, y_{1}, z_{1}\right)$ and $\left(x_{2}, y_{2}, z_{2}\right)$ are the motion path of the particle in the past time interval of $d t$ obtained by measuring. Since both $d t$ and $d r$ are very small, the measured track $d r$ has been very close to the true motion trajectory of the particle in the past. In mathematical language, the path integral $d t$ is the movement track of the particle in the past minute intervals. In a certain space, only if the past position of a particle is determined continuously, and the past momentum of the particle cannot be uncertain. If we only measure the momentum continuously, we can also get the result that the past position has definite value in a certain space. It can be seen that the uncertainty principle is not applicable to the past of microscopic particles. The same problem exists in the uncertainty relation between the energy and the action time: the energy of a photon passing through the space can be measured accurately enough by its wavelength (or frequency); according to the uncertainty relation, the measurement time must be infinite. But the actual situation is not a very long time to be able to measure the photon energy.

\subsection{Heisenberg Relationship Is Not Necessarily a Relation of Measurement Uncertainty, $\Delta x$ Is Not the Uncertainty of Position Measurement}

We can derive Heisenberg relation from the classical motion law. For the microscopic particles as bound states of uniform circular motion, the product of its curvature radius $r$ and linear momentum $p$ is equal to $\hbar$, that is, it's classic orbital angular momentum is $r p=\hbar$. Using the several relations of $r \leq \Delta x, \Delta p=p \sin \theta$ and $\sin \theta \leq 1, r p=\hbar$ can be turned into the form of $\Delta x \Delta p \geq \hbar$. The method is $r p=\hbar$ on both sides of the same times by $\sin \theta$, result in $r p \sin \theta=\hbar \sin \theta$. Because $\theta$ is the angle between the tangent and the direction of movement, always have the relations of $\sin \theta \leq 1$. So, we have $r p \sin \theta \leq \hbar$. To make $\Delta p=p \sin \theta$, we have $r \Delta p \approx$ $\hbar$. This is the formula whose shape is similar to Heisenberg relation obtained by 
according to the equation of orbit motion [25]. Note: for the regular curve motion of the particle, $\Delta p=p \sin \theta$ is not the uncertainty of the movement direction of the particles caused by random appearing in the range of $0 \rightarrow \theta$, but is the emergence value of momentum in the normal direction appear with a certain law. For uniform circular motion, it is the radial component of momentum. When a high-speed electron go through the slit consisting of two fixed atoms, the effective action distance $r$ of the electron passing through from the slit is less than or equal to the slit width $\Delta x$ (that is $r \leq \Delta x$ ). In this way, when a particle does a uniform circular motion of bound state, its $r p=\hbar$ becomes $\Delta x \Delta p \sim \hbar$. At this time, although $\Delta x$ is slit width, it is definitely not the uncertainty of position measurement. For high speed particles through the simple slit, there is a tight logical connection between $r$ and $\theta$. Once $r$ has been accurately measured, both $\theta$ and $\Delta p=p \sin \theta$ can be calculated accurately. Once $\theta$ has been accurately measured, $r$ can be accurately calculated. It is not possible that $r$ and $\theta$ only one can be accurately measured.

This brief derivation process show: If it is not assumed that the microscopic particles can't do orbital motion, Heisenberg relation cannot express that momentum and position cannot be accurately measured simultaneously. At the same time, it show that the formula whose shape is similar to Heisenberg relation doesn't deny "the state of microscopic particles can only be described by the wave function $\psi(x, t)$, and cannot be described accurately by the classical state function $f(r, p)$ ". It can be seen that the uncertainty relation itself cannot completely exclude orbital motion. The uncertainty relation can tolerate the determination and track motion of microscopic particles. Heisenberg principle has been misinterpreted by the quantum physicists in Copenhagen School. In the process of deriving the uncertainty relation, first, Heisenberg supposes that the momentum and position cannot be accurately measured simultaneously according to the intuition, $\Delta x \Delta p=\hbar$ relation formula is derived later. After that, it was interpreted as "two mechanical quantities whose operators are not commutation cannot have determined value simultaneously". This explanation obviously contains the component of the accommodation of Heisenberg hypothesis. Its logical error is the widths $\Delta x$ to be treated as the measurement uncertainty of spatial position. People also mistakenly extended the application scope of Heisenberg relation to the free movement particles and the movement particles of bound states only to have constant interference.

It has been clearly pointed out, the slit width $\Delta x$ is not a measurement uncertainty, but the reduction action distance between incident particle and nuclear. In the electron diffraction experiment, $\Delta x$ is also the artificial region of random disturbance rather than the measurement uncertainty of position. When we shoot the beyond window into the distance with a gun, the location of the bullet through the window is random, but the size of the window is not the measurement uncertainty of the bullet's position. When a plane pass through a bridge, the size of openings is not the measuring uncertainty of the position of a plane. It is similar to the case of a high speed electron passing through a slit. According 
to the theory of probability and mathematical statistics can know that the significance of $\Delta x$ in $\Delta x \Delta p \approx \hbar$ does not conform to the definition of uncertainty in spatial measurement (the method obtained $\Delta x$ is not the evaluation method for position measurement uncertainty). If we do some electron diffraction experiments in a chamber, will certainly be able to intuitively find the slit width to be not the position measurement uncertainty of high-speed electron. This kind of experiment can also judge whether the electron between the slit and the screen is in the state of diffuse (or discrete). If the electron has a clear orbit in such an experiment, the diffraction pattern can be formed, which indicates that the diffraction is not electron diffraction.

The theoretical basis for the uncertainty relation and the explanation of Copenhagen's quantum mechanics are the Von Neumann theory, the assumption of the De Broglie wave and "uncertainty relations can be derivate based on quantum mechanics basic postulate". However, he above stringent analysis shows that, these three bases are also unreliable. Since the Heisenberg relation is not necessarily the measurement uncertainty relation, and $\Delta x$ is also not the uncertainty of position measurement, then, the principle of uncertainty mathematical expression can be derived by according to the basic premise of quantum mechanics, it cannot be established as a solid foundation for the principle of uncertainty. $\Delta x \Delta p=\hbar$ is the Heisenberg relationship rather than the uncertainty relation.

Unless denying that the particle is a point particle or an entity, the particle's past can be accurately measured, whether or not it is subject to random interference. So the conclusions of this section echo each other with the conclusions of Sections 6.2 and 6.4 .

\section{The Main Content and Advantage of Tu's Measurement View and Interpretation System of Quantum Mechanics}

According to the existing quantum mechanics, the microscopic particles have a verifiable property until the particles are measured or observed in some way. At this time, a particle can also appear in two or more places. But once measured, a particle collapses into a more classical reality, and only appears in one place. This concept can lead to two problems: first, the state before the measurement can never be verified experimentally; This leads to that a number of conclusions are at the inferred level (can only be speculative, which cannot be considered to have been verified by experimental methods); Second, is the measurements led to the collapse and return to the classic reality, or is already the case (classic reality), never said clearly. There are no two problems in the measurement of quantum mechanics. There are no such problems as described in section second.

\subsection{Tu's Interpretation System of Quantum Mechanics}

The basic contents of QIMT and the measurement view and interpretation of quantum mechanics are as follows. 
1) Section 6.2 shows the structure of the object particles-an object particle is composed by a circularly polarized photon. It shows the source of the wave function of object particle (for example, an electron is not a wave packet but a monochromatic wave). The square of the absolute value of the wave function is the field strength rather than the probability density. After the discrete waves form the localized particles, their whole is no longer discrete, and will not be non-localized. The moving route of the particle is the moving path of the point particle (the field source moves with its field: according to practice, often only consider the motion of the field source, it is also the movement of the center of gravity). In the case of a hydrogen atom, the extra-nuclear electron will not reach far places. In the space point at a distance, the electromagnetic field strength is not zero rather than the appearing probability of an electron is not zero. If you want to let the object particles return to the state of discrete wave, must go through a decay process. In other words, only through a slit, the object particles will not return to the state of discrete waves.

As stated in section 6.2, the wave of the object particles is not a probability wave. An object particle of motion will not appear, which come and go like a shadow, in the whole space. Under the condition of quantum inverse measurement, the eigenvalue obtained by a measurement is the only eigenvalue of the measuring time rather than the one of many alternative eigenvalues (pure objective results can be obtained by measurement of interference-free or the interference to can be ignored). We no longer need the concept of wave collapse.

The light-knot electron structure model shows that an elementary particle is composed of a photon which twisted together. The wavelet packet structure model of the elementary particle is denied. The main contents of the existing superposition principle and wave-particle duality are also denied. The whole of the kinked photon is local and has a center of gravity, and the energy is relatively concentrated above the field source. The movement of such elementary particles is the movement of the local entity, in mechanics can be seen as the movement of point particles. The results of double slit diffraction experiments of physical particles were explained by the side effects of companion light. All phenomena of microscopic particles can be explained by the use of local realism and determinism. The uncertainty of microscopic particles can only be caused by random interference, rather than spontaneous and inherent. This explanation is Tu's interpretation of quantum mechanics.

The light-knot basic particle model tells us that, the free movement of the whole particle should not be described by wave function, and the bound motion of particles in atoms and molecules is suitable for describing by wave functions.

2) In the diffraction experiments by double-slit of the object particles, a object particle can only pass through a slit at a time. The diffraction is caused by the effect of accompanying-light, is the performance of Huygens principle, and it is not possible to prove that the particles are non-localized. The truth is similar to that the diffraction of a single water wave through a double slit cannot prove that the water is nonlocalized". The essence of Huygens principle unknown is the 
expression of the complexity of the photon rather than the overall performance of the object particles.

At the same time, a particle cannot appear in two different places. The past position and momentum of the particles can be accurately measured simultaneously.

3) There is the measurement that only the information or substance is transmitted from the measurement system to the observer. This measurement is equivalent to the measurement of the measured system without interference.

4) In the process of quantum inverse measurement, there is not the coupling between the instrument and the measured quantum system, and the particle state of non-distortion can be obtained. In the coupling between the measured particle and the instrument, the process and the results caused by instruments affect of the measured particle and by the particle to be influence of the instrument are asymmetric.

5) The Archimedes segmentation-point between the observer and the measured object can be obtained. The new measurement view in the context of QIMT is that "under certain conditions, it has to take into account the role of the instrument".

6) The non superposition state of microscopic particles can be observed by the quantum inverse measurement. Spontaneous original superposition state of microscopic particles (the superposition state between yourself and your shadow) is not the normal state of microscopic particles. That is to deny that the superposition state is the normal state of microscopic particles. Change a way of expression, the superposition of virtual states is also a virtual reality. That is, the superposition there is no filling of empty state can be carried out at any time, but there are harsh conditions for the superposition of the filled states. For instance, the superposition of atomic hollow orbits can be carried out at any time, but the superposition of the track how to fill the electron is conditional. The superposition of coherent light is easy to implement, but it is not easy to realize the superposition of electron and electron. It is conditional on whether or not the homologous conjugated particles are stacked, rather than being in a state of superposition.

The situation will not happen that the classic characteristics of a particle are lost by the superposition between the particle and its own shadow and the loss. The possibility is less likely that the twins lose their independence due to superposition of states.

7) QIMT reveals that the superposition principle can only be an assumption of states superposition. There is still a lack of conditions to lift it up to the principles. Even if it is called assumption of state superposition, it is also not universal in the micro world. The universality of quantum state superposition is denied, and the universality of quantum mechanical probability interpretation is also denied.

8) QIMT closer to local realism than quantum weak measurement theory.

9) The quantum inverse measurement can realize continuous measurement of 
the measured system without interference, and can be accurately measured everywhere. In this way, for the past of microscopic particles, it is impossible that the motion and position must not be accurately measured at the same time. It reveals that there is a logical contradiction in the principle of uncertainty.

10) The anti interference ability of quantum superposition states (quantum coherent states) is not zero, and the observer's consciousness has no effect on the state of the microscopic particles.

11) Twin particles are always pigeon pair. Wave packet collapse does not exist (even if there is a wave packet collapse, after the collapse, the state is the true colors of particles). Both quantum teleportation and quantum entanglement do not exist.

12) QIMT itself does not deny the existence of a variety of phenomena (and related theories) that instruments have a serious interference with the measured system. Only interference is random, the result is random.

\subsection{The Advantages of Tu's Measurement View of Quantum Mechanics}

Existing important explanations of quantum mechanics require or acknowledge the existence of ghosts. The theory of more worlds does not need to think of particles as ghosts. However, the division and choice of the world (the distribution of signals in different worlds) requires a ghost or God to complete. The necessary condition for the existence of the world is that different worlds can overlap each other but must be independent of each other in the affairs of the process of things. It logically denies the independence of different worlds that the same observer can see signals from different worlds. If different world cannot be independent, more worlds also does not exist (i.e., still only one world). For the explanation of the experimental phenomenon, it is very important to find out the explanation which does not need any ghost and God.

Quantum inverse measurement theory has opened up a new method for the thorough application of quantum mechanics: quantum measurement has been integrally turned into an objective physical process without subjective intervention fundamentally. The advantage of the Tu's measurement view and interpretation system of quantum mechanics lies in that it is deterministic and localrealism. It can explain all the experimental phenomena of quantum mechanics, predicted the phenomena that the previous explanation system cannot predict. They are described as follows:

(1) Can explain the source of the wave function

See Sections 6.2 and 7.1 (a) for details. Explanation of the source of the wave function is also explanation of the causes of the wave particle duality. Using the structural characteristics of particles to illustrate the nature of particles is also a major feature of this article (in this respect, Tu's theory is better than others' theory of homogeneity).

(2) Has the nature of local realism and determinism

It is assumed that the discrete waves propagate along the closed path to form 
the localized particles. The object particles can be described by wave functions, but the whole is consistent with the definition of classical particles. In the absence of decay, the object particles are localized and cannot be returned to the discrete state, and the movement can be described by coordinates and momentum. The future state of the particles can be accurately predicted. The superposition of the two object particles is generally a linear superposition of the field. The volatility of the observed object particles is actually a side effect, not a manifestation of the essential properties of the particles. There is the micro-measurement of interference-free or that the interference can be ignored. Under such a measurement condition, a purely objective state can be obtained. Only by random interference, the measurement result is random.

(3) Not contrary to common sense, not confusing, do not need the concept of wave collapse

The last advantage determines this advantage. This paper argues that: instantaneous quantum entanglement is derived from that the twin particles are the pigeon pair originally; the empirical chain of the derivation of the instantaneous quantum entanglement conclusion is incomplete; the conclusion of the continuous quantum entanglement experiment is defined by the experimenter. The interference fringe in the diffraction experiment of the object particles is the side effect caused by the accompanying-light. In this way, quantum mechanics is no longer necessary to break the common sense, it is no longer confusing.

(4) Logicality is strong, and contradictory is less

This is decided by the above three.

(5) Easy to understand, easy to learn and remember.

This is also determined by the above (1) (2) (3).

(6) Can predict the phenomena that other theories cannot predict

See Section 7.3 for details.

\subsection{The Predictions of Tu's Measurement View of Quantum Mechanics}

(1) Doing the diffraction experiment by double-slit of electron in the spark chamber or the cloud chamber, we can observe the movement track of the electron, and can determine which slit is passed through by an electron. Outside such an experimental device, if we add a magnetic field or an electric field, a small number of spots drift but the overall pattern of diffraction does not drift.

(2) If insert a piece of glass that the electron beam cannot pass through but the photon can between the phosphor screen and the slit in an ordinary electron diffraction instrument, the diffraction stripes can still be formed.

(3) If insert a piece of sheet that the photon cannot pass through but the electron can pass between the phosphor screen and slit in the ordinary instrument of electron diffraction, the first order diffraction pattern cannot be formed (Secondary diffraction pattern may appear).

(4)The diffraction can be caused by the particles from the accelerator.

It can deny the conclusion that "wave pack collapsed" can be caused by any 
experiment.

(5) Whether it is still or movement, an object particle can flip (It's like a pingpong ball and football shot out) and doesn't flip.

\section{Design of Verification Experiment of QIMT and Tu's View Measurement of Quantum Mechanics}

In summary, the main predictions of QIMT: There is the measurement method that has without interference on the measured particles, the intrinsic state of microscopic particles can be observed without damage (the 3D regression curve of charged particle tracks in cloud chamber and spark chamber is the accurate motion path of the particle); Both state superposition and coupling are conditional, free microscopic particle and partially bound microscopic particle do not overlap with their shadow, and the superposition of the homologous conjugated particles must meet the harsh conditions (state superposition is not unconditional and universal); The experimental results of quantum entanglement can be explained in this way-the quantum state is not changed when measurement of the first particle, the measured states of the twinborn second particle is not a newly formed, but it has always been like this; Uncertainty principle sometimes exists logical contradiction and is not universally applicable; Electron diffraction experiment can be explained with the accompanying-light effect. Designs in this section are in order to test these predictions to constitute a new measurement view of quantum mechanics and QIMT. In reference [26], the energy and size of more than 10 atoms and small molecules have been successfully calculated by using the quantum mechanical model of localized realism. It is another proof of the point of view in this article.

Such verification experiments must be in line with the two conditions: First, it must contain the independent process only there is information inverse transmission (that is, it must contain the process of measurement of target particles without interference, or the interference strength is less than the ability of target particles to resist interference); second, the existence or disappearance of the superposition of quantum states is easily observed.

1974, professor Pier Giorgio Merli used electron to do Yang's Interference experiment by double-slit (one of the "Which-way" experiments). In that experiment, it is only the common way that there is serious interference used by monitoring electrons [27] [28] [29]. It is necessary to replace all other monitoring methods (in particular, the monitoring methods are consistent with the conditions of quantum inverse measurement) to re-test. If the results of experiments are the same under a variety of monitoring methods, we can be sure that human consciousness can affect the behavior of electrons. Otherwise, the conclusion is too hasty. In the experiments described below, the monitoring method was changed to a cloud chamber, a spark chamber, and electrodes.

Except for particles emitted by radioactive material, artificial high-speed particles are all subjected to an accelerated process by the instrument. This acceleration process is a generalized measurement process (the process in which the 
measured particles are affected by the instrument). However, the particles coming out of the end of the accelerator still have diffraction behavior of embodying quantum properties. This indicates that the measurement (or local measurement) does not necessarily lead to collapse of the wave packet, and can meet the conditions of quantum inverse measurement. Previous experiments do not allow for further validation of this conclusion. Therefore, we designed a series of experiments in order to expediently verify the concept of quantum inverse measurement.

\subsection{The Electron Diffraction Experiment in a Cloud Chamber or a Spark Chamber}

The thickness of the cloud track is about $10^{-3} \mathrm{~mm}$ in a chamber. The distance between the two slits in the diffraction experiment by double slit is about 0.2 $\mathrm{mm}$. We do the electron diffraction experiment in the chamber and should be able to observe which slit is passed by an incident electron. If have observed which slit is passed by an incident electron, and the diffraction pattern can be observed at the same time, it is equivalent to have caught Schrödinger's cat under the quantum entanglement does not occur. If we do electron diffraction experiments in the spark chamber (or cloud chamber), or embedded in a cloud chamber between the screen and the slit in the device of an electron diffraction experiment, we are able to find out whether Schrödinger's cat can be caught in the quantum coherent state. By doing electron diffraction experiments in an applied electric field, observing the displacement and deformation of the diffraction pattern under the condition of the change of the electric field intensity, we can judge whether the diffraction is caused by the electron directly or by the accompanying light.

If the diffraction is indeed caused by the accompanying light, that the diffraction was measured could not show that the particle was in a quantum coherent state. Both the superposition principle and the concept of wave-particle duality lack of experimental basis. The principle of superposition and the concept of wave-particle duality to be not always established (to be not universally applicable) are the need and prediction of QIMT. If the electronic double-slit diffraction experiment is done in a cloud chamber or a spark chamber, the result must be one of the ones in Table 2. If the experiment is combined with an electron diffraction experiment in an applied electric field, its verification capability is stronger.

Through the analysis of the verification experiment of the quantum inverse measurement, we get another important conclusion of QIMT: Can achieve continuous inverse measurement, it does not change the quantum state of the measured particles before and after the measurement, and the obtained state is the state that measured particle is not distorted.

Electron diffraction experiments in other media are designed as follows. You can also let the electron first penetrate a very thin $(40 \mathrm{~nm})$ of silicon wafer or a layer of air, and then through a slit, do the electron diffraction experiments. If diffraction can still occur, it means that the measuring instrument does not necessarily destroy the quantum state. 
Table 2. Situation analysis of the electron diffraction experiment in the chamber or spark chamber

\begin{tabular}{|c|c|c|c|}
\hline Case & Possible observed phenomena & Problem showed by phenomenon & Verification condition for QIMT \\
\hline 1 & $\begin{array}{l}\text { Not only can observe the } \\
\text { movement track of electron, } \\
\text { and observed diffraction } \\
\text { phenomenon (and the } \\
\text { interference fringes), At the } \\
\text { same time, can be able to observe } \\
\text { which slit is passed by an } \\
\text { incident electron (an electron } \\
\text { can't pass through two slits } \\
\text { at the same time). }\end{array}$ & $\begin{array}{l}\text { The measurement action does not lead to the } \\
\text { coupling between the measured electron and } \\
\text { the instrument, and does not destroy the original } \\
\text { motion state of the measured electron. The influence } \\
\text { (interference) of the instrument on the measured } \\
\text { electron can be ignored (the ability to resist } \\
\text { interference of quantum superposition states } \\
\text { is not zero). In short, such measurements } \\
\text { did not lead to the collapse of the wave } \\
\text { packet and the quantum decoherence. } \\
\text { The observation result that an electron can't pass } \\
\text { that the diffraction to have interference fringes is c } \\
\text { superposition principle and wave-particle duality are }\end{array}$ & $\begin{array}{l}\text { No interference measurement can } \\
\text { be realized. Namely, there is the observation } \\
\text { of non-distortion (there is quantum inverse } \\
\text { measurement). Both the process and } \\
\text { results that the instrument affects the } \\
\text { particle are asymmetry with the one of } \\
\text { the particle is influence on apparatus. }\end{array}$ \\
\hline 2 & $\begin{array}{l}\text { The movement track of the } \\
\text { electron can be observed, } \\
\text { but any diffraction phenomenon } \\
\text { cannot be observed. }\end{array}$ & $\begin{array}{l}\text { Using a cloud chamber to measure a } \\
\text { moving electron, destroyed the original state of } \\
\text { motion of the electron, and led to the collapse } \\
\text { of the wave packet and quantum decoherence. }\end{array}$ & $\begin{array}{l}\text { The effect of cloud chamber on the } \\
\text { measured electron is not zero. The } \\
\text { measurement by using the cloud chamber } \\
\text { cannot be used to validate QIMT. }\end{array}$ \\
\hline 3 & $\begin{array}{l}\text { The diffraction phenomenon } \\
\text { can be observed, but any } \\
\text { movement track of the } \\
\text { electron cannot be observed. }\end{array}$ & $\begin{array}{l}\text { This does not accord with the function of the } \\
\text { chamber. If this is the case, then the electrons } \\
\text { are really turned into the things of superposition } \\
\text { state of non-wave and non-particle. }\end{array}$ & $\begin{array}{l}\text { The experiment using cloud chamber to } \\
\text { capture charged particles is not in line with the } \\
\text { quantum inverse measurement conditions. }\end{array}$ \\
\hline
\end{tabular}

Note: let the electrons one by one pass through the double slit, the observation of the occurrence of the phenomenon.

\subsection{A Longitudinal Electrode or Magnet Is Mounted at the Exit Side of the Slit}

Even if the micro-particles can understand the people's consciousness, it will not have the corresponding change before the human consciousness acting it. Based on this concept, we consider observing after that the particles pass through the double-slit.

In 1897, in order to test the properties of cathode rays, Joseph John Thomson made a Crookes' cathode ray tube, and installed a pair of metal electrodes $D$ and $E$ in the middle of it. The author used this method, but installed an easily mobile magnet or a pair of electrodes in the exit-end of the electronic diffraction apparatus. The magnetic field and electric field can offset the cathode ray and lead to it not deflecting light. A comparison of the diffraction pattern of two cases, one with an added vertical electromagnetic field and one without it, can lead to the judgment that the diffraction is caused directly by the electron itself or by the accompanying light.

\subsection{Covering the Screen with a Piece of Glass, Which a Photon Can Penetrate but an Electron Cannot, in Front of the Screen}

Insert the glass to conduct the experiment: if the diffraction pattern appears only on the glass, but does not appear on the screen behind, this result indicates that the diffraction is directly caused by the undulatory property of electrons; alternatively if the diffraction pattern appears only on the screen behind, this shows the diffraction is caused by the accompanying light. 


\subsection{Covering the Screen with a Piece of Metal Foil, Which an Electron Can Penetrate but a Photon Cannot, in Front of the Screen}

Insert the metal foil to conduct the experiment: if the diffraction pattern appears only on the metal foil, but does not appear on the behind screen, this shows that the diffraction is caused by accompanying light; alternatively if the diffraction pattern appears only on the behind screen, this result shows that the diffraction is directly caused by the undulatory property of an electron.

\subsection{Use a Book of Metal Electrodes to Separate the Exit Space by a Double Slit}

In 1974, the Italian physicists Pier Giorgio Merli, Gian Franco Missiroli, and Giulio Pozzi repeated the experiment using single electrons and biprism (instead of slits), showing that each electron interferes with itself as predicted by quantum theory [27]. In 2002, the single-electron version of the experiment was voted "the most beautiful experiment" by readers of Physics World.

The experiment to have designed by me here is to use the principle that "the current interference does not affect the past experience of the particles" and observing the situation when the electrons pass through the slits. Compared with the previous experiments of electrons crossing double slits, the situation is exactly the same when the electron beam is incident on the double slits.

Just after the electron beam passes through the slit is not the same. It speculates electrons are incident and pass through which slit by means of measuring electron through which slit.

This experiment and the electron diffraction experiment in the spark chamber can confirm each other.

With certain practices, such as that shown in Figure 2, the inserted metal foil is too narrow to act as the cathode of an electrode, and the two sides of the metal foil act as the mounted anode. The distance between the two slits is generally $0.2 \mathrm{~mm}$, The middle electrode can be used with a thickness of no more than $0.2 \mathrm{~mm}$ of a sheet. Let the electrons pass through the double slit virtually one by one. Subsequently, if the electrons pass through the left slit, it will deflect to the left; alternatively if the electrons pass through the right slit, it will deflect to the right. As long as the electrode is short enough, the diffraction is caused by the

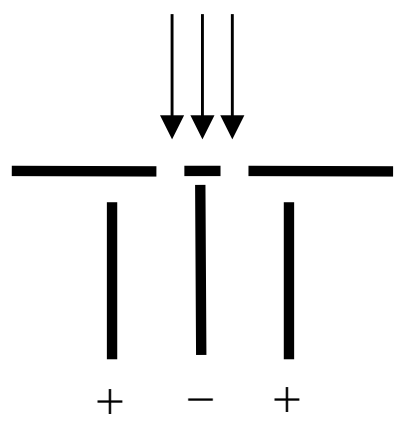

Figure 2. A double slit electron diffraction device with an electrode separator. The electrode is only a piece of plate, and the anode is the two plates together with a wire. 
accompanying light, residual diffraction should also be observed. If the diffraction is a direct result of the electron itself, the diffraction pattern cannot be observed (or the diffraction fringe of serious distortion can be observed). To distinguish which side an electron specifically passes through can also deny the phenomena of electronic volatility, thereby denying that diffraction can be caused by the fluctuation of an electron. This experiment can prove whether an electron changes its direction and become a point particle at the moment arrived in the screen. Don't think these experiments are simple. In fact, they can be used to verify QIMT and test the view of measurement and interpretation system of existing quantum mechanics.

The authors do not have the ability to do the experiments of my own design. The acute sub reader can immediately test (for example, take a shoe shaped magnet act on an existing electron diffraction instrument, and see if it can cause the deformation of the diffraction fringe). The readers who can apply to the funds can be carefully done the experiments designed by this paper. No matter how the experiment results, it is of great significance: If the experimental results deny QIMT, provided more and more evidence for the principle of superposition, the uncertainty principle and the concept of wave-particle duality and is conducive to the elimination of the existing quantum mechanics of doubt, to reduce unnecessary controversy; if the experimental results confirm QIMT, would subvert the measurement and interpretation system of existing quantum mechanics.

\section{Concluding Remarks}

The error in the interpretation and understanding of quantum mechanics stems from the lack of knowledge of the structure of the elementary particles. In addition to the limitations of this knowledge, the weakness of human nature is also the wrong source of quantum mechanics (for their own interests and they blindly follow the authority and the mainstream and suppress new ideas). The weakness of human nature leads to the mainstream scholars in interest community not to explain experimental phenomena according to facts, but to select some interpretations of experimental phenomena of quantum mechanics according to their own needs (that is, they deviated from the dialectical track and lost the logic principle because of the weakness of human nature). The most representative of the two types of wrong behavior is as follows: Strictly speaking, no Bell experiment can exclude all conceivable local-realist theories, because it is fundamentally impossible to prove when and where free random input bits and output values came into existence [30].

The diffraction of object particles is most likely caused by accompanying light. However, in case of the absence of an experimental approach to deny that the diffraction of object particles is caused by accompanying-light, orthodox physicists have used interpretations that object particles themselves have the volatility.

As long as we stick to the principle that empirical chain must be complete, and use the side effect caused by the accompanying light to explain the experimental 
results of electron diffraction, everything will change. The essence of the instantaneous quantum entanglement experiment is that the twin particles are pigeon pair before to be measured. The experimental results of the continuous quantum entanglement result from the experimenter's definition of quantum entanglement. Orthodox physicists did not use the experimental method to deny that the twin particles were originally pigeon pair. In the case of the absence of an experimental approach to deny that the diffraction of object particles is caused by accompanying light, the orthodox physicists have used interpretation that object particles themselves have the volatility. It can be seen that the conclusions in this paper are no harsher than the previous physicists. See Section 7.2 for additional information on Tu's theory.

Before the prophecy is not verified, the existing phenomena have been explained by means of that do not violate the common sense, and the logically self-consistent have been done. This reflects the significance of this research work (there is the value of discussion and hot debate). Once the prophecies have been experimentally verified, it will quickly change the concept of the people understanding the micro-world, end the debate on quantum mechanics, and so that quantum theory and its application back to the correct direction of development. The birth of the interpretation program of quantum mechanics for local-realism and determinism also opens the revolutionary path for material structure theory.

Quantum inverse measurement theory has created a new method of thorough application of quantum mechanics-Quantum measurement has been turned, from the whole, into an objective physical process without subjective intervention. Tu's interpretation of quantum mechanics is at least an alternative quantum mechanical interpretation scheme. It also needs to be supplemented and perfected. The most urgent task is to complete the verification experiments designed in this paper. Tu's measurement view and interpretation system of quantum mechanics must be applied to quantum electrodynamics. The relation between the Tu's interpretation system of quantum mechanics and the mathematical formalism of quantum theory must also be established. The mutual transformation between photonics and electrons, photons and protons has been a reliable experimental evidence. However, the mechanism of such a transformation process remains to be studied. I hope the conditional readers will do it voluntarily. It is necessary to verify the predictions in this paper by using the experimental method, even if it is to maintain the old quantum mechanics interpretation system.

\section{Acknowledgements}

Thanks to my family for their long-term support and encouragement!

\section{References}

[1] Tu, R.S. (2015) Local Realism Quantum Mechanics. American Academic Press, Lanham, 1-242. 
[2] Aharonov, Y., Albert, D.Z. and Vaidman, L. (1988) Physical Review Letters, 60, 1351. https://doi.org/10.1103/PhysRevLett.60.1351

[3] Turck, Y. (2012) Quantum Weak Measurement Theory and Its Applications. Graduate University of Chinese Academy of Sciences.

[4] Rozema, L.A., Darabi, A., Mahler, D.H., Hayat, A., Soudagar, Y. and Steinberg, A.M. (2012) Physical Review Letters, 109, 100404.

https://doi.org/10.1103/PhysRevLett.109.100404

[5] Kocsis, S., Steinberg, A.M., et al. (2011) Science, 332, 1170. https://doi.org/10.1126/science.1202218

[6] Einstein, A. (1936) Journal of the Franklin Institute, 221, 313-347. (Reprinted in Translation in Einstein 1954)

[7] Einstein, A., Podolsky, B. and Rosen, N. (1935) Physical Review, 47, 777-780. https://doi.org/10.1103/PhysRev.47.777

[8] Weinberg, S. (2017) The Trouble with Quantum Mechanics. The New York Review of Books, January 19, 2017 Issue.

http://www.nybooks.com/articles/2017/01/19/trouble-with-quantum-mechanics/

[9] Liu, Z.H. (2016) Bizarre: What Kind of Microscopic Mystery Does the Upcoming Quantum Emission Satellite Want to Explore? 360doc. Personal library. http://www.360doc.com/content/16/0815/08/27398134_583297386.shtml

[10] Shi, Y. (2017) Continue the Quantum Mechanics Revolution. Guangming Daily, May 25, 2017. 13th Edition.

[11] Fock, B.A. (1968) Natural Dialectics Research, 3, 42-46.

[12] Bohr, N. (1929) The Quantum of Action and the Description of Nature. Naturwissenschaften. Planck sp\$ecial iss\$ue. (Originally published in German in Naturwissenschaft, Vol. 17, 1929. First published in English in 1934 by Cambridge University Press.)

[13] Sakurai, J.J. and Jim, J. (2010) Modern Quantum Mechanics. 2nd Edition, Pearson Education Limited, 223-241.

[14] Cohen, D.W. (1989) An Introduction to Hilbert Space and Quantum Logic. Springer-Verlag, 94-104. https://doi.org/10.1007/978-1-4613-8841-8

[15] Bohr, N. (1935) Physical Review, 48, 696. https://doi.org/10.1103/PhysRev.48.696

[16] Leibfried, D., Knill, E., Seidelin, S., Britton, J., Blakestad, R.B., Chiaverini, J., Hume, D.B., Itano, W.M., Jost, J.D., Langer, C., Ozeri, R., Reichle, R. and Wineland, D.J. (2005) Nature, 438, 639-642. https://doi.org/10.1038/nature04251

[17] Häffner, H., Hänsel, W., Roos, C.F., Benhelm, J., Chek-al-kar, D., Chwalla, M., Körber, T., Rapol, U.D., Riebe, M., Schmidt, P.O., Becher, C., Gühne, O., Dür, W. and Blatt, R. (2005) Nature, 438, 643-646. https://doi.org/10.1038/nature04279

[18] Wang, Y.-T., Tang, J.-S., Wei, Z.-Y., Yu, S., Ke, Z.-J., Xu, X.-Y., Li, C.-F. and Guo, G.-C. (2017) Physical Review Letters, 118, 020403.

[19] Hensen, B., Bernien, H., Dréau, A.E., Reiserer, A., Kalb, N., Blok, M.S., Ruitenberg, J., Vermeulen, R.F.L., Schouten, R.N., Abellán, C., Amaya, W., Pruneri, V., Mitchell, M.W., Markham, M., Twitchen, D.J., Elkouss, D., Wehner, S., Taminiau, T.H. and Hanson, R. (2015) Nature, 526, 682-686. https://doi.org/10.1038/nature15759

[20] Schrödinger, E. (1935) Proceedings of the Cambridge Philosophical Society, 31, 555-763.

[21] Von Neumann, J. (1955) Mathematical Foundations of Quantum Mechanics. Princeton University Press, Princeton, Germany Version 1932. 
[22] Bell, J.S. (1964) Physics, 1, 195-200.

[23] Aspect, A., Grangier, P. and Roger, G. (1981) Physical Review Letters, 47, 460.

[24] Kasday, L.R., Ulman, J.D. and Wu, C.S. (1975) Angular Correlation of ComptonScattered Annihilation Photonsand Hidden Variables. Nuovo Cimento, 25B, 633661.

[25] Tu, R.S. (2013) Infinite Energy, No. 107, 44-46. http://www.docin.com/p-765650007.html

[26] Tu, R.S. (2014) Journal of Modern Physics, 5, 309-318. https://doi.org/10.4236/jmp.2014.56041

[27] Meili, P.G., Missiroli, G.F. and Pozzi, G. (1976) American Journal of Physics, 44, 306-307. https://doi.org/10.1119/1.10184

[28] Rosa, R. (2012) Physics in Perspective, 14, 178-195. https://doi.org/10.1007/s00016-011-0079-0

[29] Kocsis, S., Braverman, B., Ravets, S., Stevens, M.J., Mirin, R.P., Shalm, L.K. and Steinberg, A.M. (2011) Science, 332, 1170-1173. https://doi.org/10.1126/science.1202218

[30] Hensen, B., et al. (2015) Nature, 526, 682. https://doi.org/10.1038/nature15759

Submit or recommend next manuscript to SCIRP and we will provide best service for you:

Accepting pre-submission inquiries through Email, Facebook, LinkedIn, Twitter, etc. A wide selection of journals (inclusive of 9 subjects, more than 200 journals) Providing 24-hour high-quality service User-friendly online submission system Fair and swift peer-review system Efficient typesetting and proofreading procedure Display of the result of downloads and visits, as well as the number of cited articles Maximum dissemination of your research work

Submit your manuscript at: http://papersubmission.scirp.org/ Or contact jmp@scirp.org 